\title{
Dynamics of intramolecular vibrational-energy redistribution (IVR). II. Excess energy dependence
}

\author{
Peter M. Felkera) and Ahmed H. Zewail ${ }^{\text {b) }}$ \\ Arthur Amos Noyes Laboratory of Chemical Physics, ") California Institute of Technology, Pasadena, California 91125
}

(Received 9 November 1984; accepted 17 December 1984)

The results of picosecond-resolved measurements of intramolecular vibrationalenergy redistribution (IVR) in jet-cooled anthracene at different excess energies are presented. From these results, the nature of IVR as a function of vibrational energy, the relevant time scales for the process, and the details of pertinent vibrational couplings are determined.

\section{INTRODUCTION}

In the context of the theory presented in the previous paper (I) this paper provides an account of experimental results pertaining to IVR in anthracene. The results were obtained with temporal resolution five times better than that used in previous studies. ${ }^{1-3}$ This has allowed the resolution of beat frequencies up to $\sim 12 \mathrm{GHz}$, and the resolution of decays with time constants on the order of 10 ps. A primary emphasis of the work is on elucidating the changes which take place in IVR processes as the vibrational energy of the molecule increases. In addition, using the theoretical results of I on multilevel systems we fully analyze our experimental data and obtain parameters associated with the Hamiltonian matrix for vibrational coupling in anthracene. This is done for excess vibrational energies ranging from 0 to $\sim 2000 \mathrm{~cm}^{-1}$.

The organization of the paper is as follows. Firstly, the experimental techniques and data analysis are discussed. Of principal importance in this section is the presentation of the Fourier analysis methods which allow one to extract quantum beat frequencies, phases, and modulation depths from fluorescence decays. Secondly, the results for various single vibronic levels (SVL's) in $S_{1}$ anthracene are presented in the order of increasing SVL energy. These results are analyzed to determine the number of coupled levels giving rise to the observed behavior and, where possible, to dètermine elements of the Hamiltonian matrix in the zero-order vibrational basis set. Finally, the overall trends of the results and the timescales for IVR in the molecule are discussed.

\section{EXPERIMENTAL}

\section{A. Apparatus}

The picosecond-jet apparatus used in the experiments reported herein is described in detail elsewhere. ${ }^{2}$ The nozzle conditions were much the same as for previous work on anthracene. ${ }^{1-5}$ The anthracene sample (Aldrich gold label, $99.9 \%$ ) was heated to $\sim 180^{\circ} \mathrm{C}$ and expanded through a $\sim 100 \mu \mathrm{m}$ pinhole with $30-40$ psi helium. The

\footnotetext{
a) IBM Graduate Fellow.

b) Camille and Henry Dreyfus Foundation Teacher-Scholar.

c) Contribution No. 7117.
}

laser-to-nozzle distance was typically $3 \mathrm{~mm}$. The excitation source consisted of the frequency-doubled $\left(\mathrm{LiIO}_{3}\right)$ output of a synchronously pumped, cavity-dumped dye laser (DCM as dye), the bandwidth of which was $\sim 2 \mathrm{~cm}^{-1}$ FWHM and the temporal pulse width of which was 15 ps FWHM. Fluorescence was collected with right angle geometry and directed through a $0.5 \mathrm{M}$ monochromator. The dispersed fluorescence spectral resolution varied with the particular measurement being made and is noted with the data. Fluorescence photons were detected with a Hamamatsu R1564U multichannel plate photomultiplier, the output of which was amplified before encountering the timing electronics. Decays were measured using timecorrelated single photon counting. The total temporal response of detection (response function) was typically 80 ps FWHM as measured by scattering laser pulses off of the nozzle. Care was taken to monitor any major temporal shifts in the peak of the response function since such shifts affect the apparent phases of quantum beat components. Generally, large shifts were not a problem and are not relevant to any of the results included herein.

\section{B. Extraction of beat parameters by Fourier analysis}

It is well known that a measured fluorescence decay $F(t)$ may be related to a true molecular decay $I(t)$ by the convolution integral ${ }^{6}$

$$
F(t)=\int_{0}^{t} R\left(t^{\prime}\right) I\left(t-t^{\prime}\right) d t^{\prime}
$$

where $R(t)$ is the response function of the experimental apparatus. From theoretical considerations ${ }^{7}$ pertaining to vibrational quantum beats the molecular decays are expected to be of the form

$$
I(t)=\operatorname{Re}\left[A e^{-\Gamma t}\left(1+\sum_{I>J=1}^{N} M\left(\omega_{I J}\right) e^{-i \omega_{I J} t}\right)\right],
$$

where $A$ is a constant, $\Gamma$ is the excited state decay rate, $N$ is the number of coupled vibrational levels, and $M\left(\omega_{I J}\right)$ is the modulation depth of the beat frequency $\omega_{I J}$. Now, since one is interested in extracting the values for $\omega_{I J}$ and $M\left(\omega_{I J}\right)$ from a measured decay, Fourier analysis is the natural method by which to accomplish this. In particular, 
one can make use of the theorem ${ }^{8}$ for the Fourier transform of a convolution integral to obtain

$$
F(\omega)=I(\omega) R(\omega)
$$

or

$$
I(\omega)=F(\omega) / R(\omega),
$$

where $F(\omega), I(\omega)$, and $R(\omega)$ are the Fourier transforms of $F(t), I(t)$, and $R(t)$, respectively. This procedure of dividing the transform of the measured decay by the transform of the response function automatically compensates for two defects in $F(\omega)$ : (1) it enhances the amplitudes of the higher frequency components which tend to be washed out by the finite response function width and (2) it establishes the proper phases for the beat components. By measuring $F(t)$ and $R(t)$, and then calculating $F(\omega)$ and $R(\omega)$, one can obtain $I(\omega)$ by Eq. (2.4). For this paper we have calculated $F(\omega)$ and $R(\omega)$ by using the CooleyTukey fast Fourier transform algorithm ${ }^{9}$ to transform data zero filled ${ }^{10}$ to 4096 points.

After finding $I(\omega)$, the $\omega_{I J}$ are easily obtained by the positions of the bands in the Fourier spectrum. The values of the beat frequencies reported herein are actually the values $\omega_{I J} / 2 \pi$. The accuracy of these numbers is ultimately limited by the resolution of the Fourier spectra and the accuracy of the time base. A conservative estimate of the error in quoted beat frequencies is $\pm 5 \%$.

The $M\left(\omega_{I J}\right)$ are available from the Fourier amplitudes of components in $I(\omega)$, as is evident from

$$
\operatorname{Re}[I(\omega)]=K\left[\frac{\Gamma}{\Gamma^{2}+\omega^{2}}+\sum_{D J=1}^{N} \frac{\Gamma M\left(\omega_{I J}\right)}{\Gamma^{2}+\left(\omega_{I J}-\omega\right)^{2}}\right] .
$$

In principle, the heights of the bands of $\operatorname{Re}[I(\omega)]$ normalized to the zero-frequency band height should give the various absolute modulation depths $M\left(\omega_{I J}\right)$. In practice, it is more accurate to integrate over the individual band shapes in $I(\omega)$ and to then normalize these sums to the integrated intensity of the zero-frequency band. This is better for two reasons. Firstly, small variations in vibrational coupling with rotational level ${ }^{11}$ tend to broaden the nonzero-frequency bands in $I(\omega)$ relative to the breadth $\Gamma$ of the zero-frequency band. That which is pertinent, therefore, are not the heights but the integrated intensities of the bands. Secondly, it is often necessary to collect data in such a manner that decays are truncated at a significant fraction of their initial intensities. This has the effect of introducing sinusoidal oscillations about zero into the derived $I(\omega)$. Summing over a band averages out these oscillations and, by so doing, gives a more accurate value for the Fourier amplitude.

A number of factors contribute to errors in the derived values for the modulation depths (see Sec. IV of I). The first is noise in the Fourier spectra. The second factor arises from the inability to completely spectrally isolate bands in the fluorescence spectrum. The influence of this factor varies from band to band. It can be significant even for detection resolution better than $1 \AA$. Thirdly, small drifts in the response function during the course of the measurement of a decay can make a large difference in the values derived for the modulation depths of high frequency beat components. This is the case because the width of the measured response function $R(t)$ will, in general, be narrower than the effective response function $R_{\mathrm{eff}}(t)$ corresponding to the decay. [Typically, the time needed to measure a response function is a small fraction of the time needed to measure a decay. This is why one expects $R(t)$ to be narrower than $\left.R_{\text {eff }}(t)\right]$. Thus, in Eq. (2.4) $R(\omega)$ will be larger than it should be. A specific example assuming a Gaussian response function will illustrate the point. If the measured $R(t)$ is 77 ps FWHM, while $R_{\mathrm{eff}}(t)$ is 85 ps FWHM (only a drift of one channel under typical experimental conditions) then $R(\omega)$ $\simeq R_{\text {eff }}(\omega)=0.975$ for $\omega / 2 \pi=1 \mathrm{GHz}$, but $R(\omega)=0.218$ while $R_{\text {eff }}(\omega)=0.158$ for $\omega / 2 \pi=8.5 \mathrm{GHz}$. By using $R(\omega)$ in Eq. (2.4) essentially no error will be introduced for the $1 \mathrm{GHz}$ modulation depth, but the modulation depth derived for the $8.5 \mathrm{GHz}$ component will be only $0.158 /$ $0.218=72 \%$ of its actual value. One expects this source of error in modulation depths to increase in influence as the time needed to measure a decay increases. It is likely that it is the major source of error in the quoted modulation depths of high frequency components.

Despite the value that $I(\omega)$ has in the analysis of a beat-modulated fluorescence decay, the large zero-frequency component and the marked, truncation-induced oscillations of a typical $I(\omega)$ spectrum render it relatively unattractive for display purposes. On the other hand, the Fourier spectrum $I_{m}(\omega)$ derived solely from the transformation of the modulated portion of a decay multiplied by a suitable shaping function provides almost as much information as the corresponding $I(\omega)$ [absolute modulation depths are not available from $I_{m}(\omega)$ ] but is rid of the zero-frequency peak and the spurious oscillations. For this reason most of the Fourier spectra which appear as figures in this paper are $I_{m}(\omega)$ and not $I(\omega)$ spectra.

To obtain $I_{m}(\omega)$ for a given decay, the decay is first fit to a single exponential function by using a nonlinear least squares fitting algorithm. ${ }^{12}$ Convolution effects are not considered in this initial portion of the analysis since, in general, $1 / \Gamma$ is much greater than the width of $R(t)$. The fit function is then subtracted from $F(t)$ and the residual $F_{m}(t)$ is multiplied by the function ${ }^{13}\left(t_{f}-t\right)^{0.5}$, which causes it to go to zero smoothly as $t \rightarrow t_{f}, t_{f}$ corresponding to the point in time at which $F(t)$ is truncated. This manipulation substantially eliminates any spurious oscillations in the Fourier spectrum. The function $\left(t_{f}-t\right)^{0.5} F_{m}(t)$ is then Fourier transformed and divided by $R(\omega)$ to give $I_{m}(\omega)$. Now, as we have stated, $\operatorname{Re}\left[I_{m}(\omega)\right]$ preserves the phase behavior and the relative values of the $M\left(\omega_{I J}\right)$ of a given decay. One should, however, note two points about the spectra. Firstly, the application of the shaping function $\left(t_{f}-t\right)^{0.5}$ to $F_{m}(t)$ results in artificial widths for the Fourier bands in $I_{m}(\omega)$. Secondly, the shapes of the bands appearing in $I_{m}(\omega)$ depend on that portion of $F(t)$ which is chosen initially to be fit to the exponential function. The band shape distortions that arise from this second cause become more serious, the farther a decay is fit from its peak. For all practical purposes neither of the above artifacts is important enough 
to substantially modify the values for the beat frequencies, phase behavior, or relative modulation depths derived for a given decay.

\section{Analysis of fluorescence decays in the dissipative regime}

In the limit where the number of coupled vibrational levels is large enough to give rise to dissipative IVR, ${ }^{5}$ the utility of Fourier transform techniques is limited due to the large number of beat components in any given decay. For such cases, the extraction of IVR parameters from decay measurements can be accomplished by using Eqs. (5.7) and (5.8) of paper $\mathrm{I}^{7}$ to fit observed decays. Experimentally, it is easier to obtain a "pure" a-type (vibrationally unrelaxed) decay than it is to obtain vibrationally relaxed fluorescence that is uncontaminated by $a$-type emission. Thus, only fits to a-type [i.e., Eq. (5.7) of I] dissipative decays are reported herein. These fits were accomplished by the method of nonlinear least squares. ${ }^{12}$ The convolution of the true molecular decay with the temporal response of the detection system was taken into account.

\section{LOW ENERGY REGION}

The fluorescence spectroscopy of anthracene SVL's having energies from $E_{\mathrm{vib}}=0-1200 \mathrm{~cm}^{-1}$ has already been reported on. ${ }^{14,15}$ Almost all of the spectra consist of distinct bands which can be assigned exclusively in terms of transitions arising from an optically active SVL (see Sec. II $D$ of $\mathbf{I}^{7}$ for what we mean by an optically active level). These spectral characteristics are consistent with the manifestations of uncoupled vibrational levels. Previous studies in this laboratory have corroborated this interpretation in that the fluorescence decays of each of the bands in a given spectrum have been found to be identical single exponentials. These former studies, however, were done with a detection temporal resolution that only allowed beat frequencies of less than $\sim 2 \mathrm{GHz}$ to be resolved. It is now apparent that in most cases beats arising from vibrational couplings in anthracene are of frequencies greater than this. ${ }^{4}$ Thus, we have remeasured with better time resolution some decays corresponding to excitation of SVL's in the low energy regime. Figure 1 provides an example of the fluorescence spectrum and decay upon excitation of the $12^{2}\left(766 \mathrm{~cm}^{-1}\right) \mathrm{SVL}$. The spectrum is analyzed in Ref. 14. The decay, which corresponds to detection of the $390 \mathrm{~cm}^{-1}$ fluorescence band, is clearly unmodulated, and is the same as the decays of the other bands in the spectrum. (The apparent rise time of the decay is a convolution effect, as has been verified by calculating the convolution of an $18 \mathrm{~ns}$ exponential with our measured system response function. Such apparent rise times become longer, the longer the fluorescence lifetime becomes.) Decays have also been measured for other excitation bands in this low energy regime. No beats have been observed despite the temporal resolution. Both the spectral ${ }^{14,15}$ and decay results indicate, therefore, that vibrational coupling leading to IVR does not occur to an appreciable extent in anthracene SVL's
$E_{\mathrm{vib}}=766 \mathrm{~cm}^{-1}$

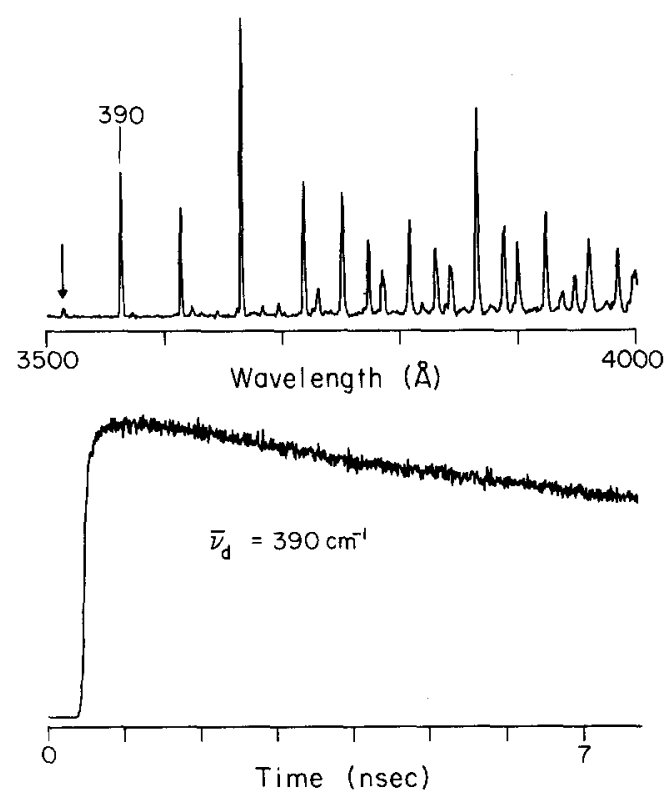

FIG. 1. Dispersed fluorescence spectrum and fluorescence decay resulting from excitation of jet-cooled anthracene to $S_{1}+766 \mathrm{~cm}^{-1}\left(12^{2}\right)$. The fluorescence spectrum was obtained with $1.6 \AA$ monochromator resolution $(\equiv R)$. An arrow marks the excitation wavelength. The decay corresponds to detection of the $390 \mathrm{~cm}^{-1}$ band in the spectrum with $R=3.2 \AA$.

up to $\sim 1200 \mathrm{~cm}^{-1}$. In saying this we do not exclude the possibility that strong Fermi resonances, resulting in splittings of several wave numbers or more, are not present in this energy regime. Indeed, other results ${ }^{15,16}$ have provided evidence for such coupling in the higher energy portion of the region. The difficulty in preparing such coupled systems coherently, however, argues against considering them in terms of dynamical energy flow. More discussion on this point is presented in Sec. VI C to follow.

\section{INTERMEDIATE ENERGY REGION}

Without exception, the excitation of jet-cooled anthracene at each of the five prominent vibronic bands in its excitation spectrum ${ }^{14,15}$ between $S_{1}+1380 \mathrm{~cm}^{-1}$ and $S_{1}+1514 \mathrm{~cm}^{-1}$ results in quantum beat-modulated fluorescence decays, the beat patterns of which depend on the particular fluorescence band being detected. We have already reported ${ }^{4}$ preliminary results for two of these excitation bands: $E_{\text {vib }}=1380$ and $1420 \mathrm{~cm}^{-1}$. In this section we analyze in detail the results for these two bands in terms of the theory presented in I, and obtain parameters pertaining to IVR and vibrational coupling at these energies. For the three other bands, we present representative decays, but do not provide any extensive analysis of the results.

\section{A. $E_{\text {vib }}=1380 \mathrm{~cm}^{-1}$ : The $a_{g}(6)$ mode}

We have previously reported results ${ }^{1,2}$ pertaining to the characteristics of the quantum beats which arise from 
excitation of this prominent band in the excitation spectrum. (The nature of the vibrational level has been assigned in Ref. 14.) In fact, it is this excitation which gives rise to those beats which we reported on three years ago and which represent the first example of a quantum interference effect in a large molecule. It turns out that these "old" results actually represent manifestations of only a minor aspect of the vibrational dynamics associated with this excitation energy; increased temporal resolution has revealed that fast beats ${ }^{4}$ modulate many of the dispersed fluorescence bands which appear to be unmodulated under 400 ps resolution. As shall be seen below, the new data lend themselves to a relatively straightforward interpretation in terms of IVR.

\section{Results}

The dispersed fluorescence spectrum resulting from excitation to $S_{1}+1380 \mathrm{~cm}^{-1}$ is shown in Fig. 2 along with a higher resolution scan of a portion of the spectrum. A previous attempt ${ }^{15}$ at assigning this spectrum made note of the fact that while a number of the bands are assignable in terms of optically active intervals (most notably those bands at shifts of $390,755,780,1168$, and $1408 \mathrm{~cm}^{-1}$ from the excitation energy), most of the bands, including some of the most intense ones, are not. This mix of assignable (vibrationally unrelaxed) and unassignable (vibrationally relaxed) bands, and the congestion in and to the red of the $S_{1}$ origin region indicate (see Sec. II $D$ of I) that the spectrum arises from a relatively small number of coupled vibrational levels.
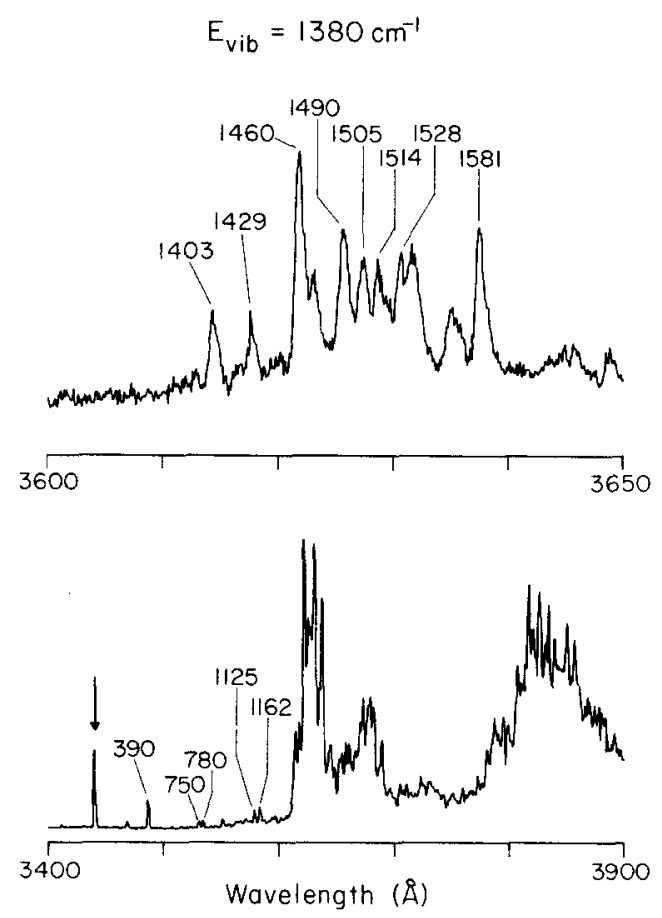

FIG. 2. Dispersed fluorescence spectra resulting from excitation of jetcooled anthracene to $S_{1}+1380 \mathrm{~cm}^{-1}$. The upper portion is a high resolution $(R=0.5 \AA)$ trace of the region in and about the wavelength of the $0_{0}^{0}$ transition of the molecule. The lower portion, taken with $R$ $=1.6 \AA$ covers a wider range and includes the excitation wavelength (arrow). Various bands in the spectra are marked with their shifts in $\mathrm{cm}^{-1}$ from the excitation energy.

$$
E_{v i b}=1380 \mathrm{~cm}^{-1}
$$

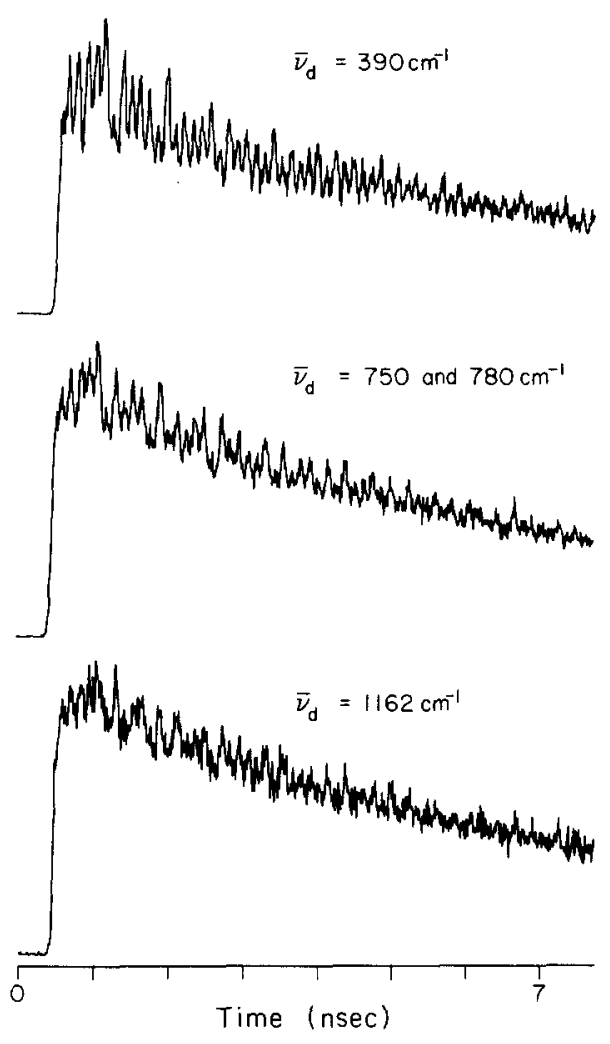

FIG. 3. Fluorescence decays of group I bands (see the text) in the $E_{\text {vib }}$ $=1380 \mathrm{~cm}^{-1}$ spectrum of anthracene. The shifts of the bands from the excitation energy are given in the figure. Being that these bands are relatively isolated spectrally, low detection resolution was used to maximize the signal. From top to bottom $R=24,16$, and $5 \AA$.

Confirmation of this is provided by time-resolved results, which reveal beat-modulated fluorescence decays that depend on detection wavelength. It is convenient to consider the decays of various groups of these bands separately.

Figure 3 presents typical decays of some of those bands (group I) in the spectrum that can be readily assigned. All of the decays have a similar appearance and have roughly the same Fourier spectra. Figure 4 (top), which is the Fourier spectrum that results from transformation of the decay of the $390 \mathrm{~cm}^{-1}$ band, is representative of these spectra. In this spectrum, eight beat components are evident at $0.2,1.8,2.1,2.6,3.5,4.3,4.9$, and 8.4 $\mathrm{GHz}$. Note that all of these components have positive phases. The Fourier spectra of Figs. 4 (middle) and 4 (bottom) illustrate the changes that take place in the 390 $\mathrm{cm}^{-1}$ decay as the excitation source is tuned $\sim 2 \mathrm{~cm}^{-1}$ to the red and to the blue of the excitation peak, respectively. Clearly, there is an enhancement in the components at $1.8,2.6$, and $4.3 \mathrm{GHz}$ for the red excitation relative to the blue. On the other hand, the blue excitation results in a decay which is almost exclusively modulated by the $3.5,4.9$, and $8.4 \mathrm{GHz}$ components (with some contribution from the $2.1 \mathrm{GHz}$ component). For blue excitation one obtains absolute modulation depths of $0.12,0.16$, and 
o) $E_{\text {vib }}=1380 \mathrm{~cm}^{-1}$
$\tilde{\nu}_{0}=390 \mathrm{~cm}^{-1}$

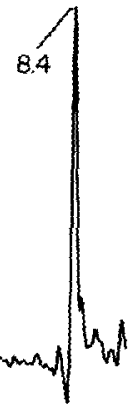

b)

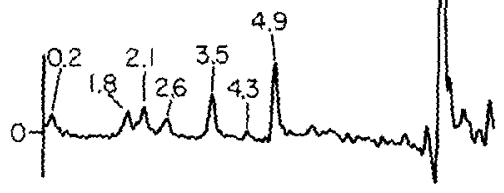

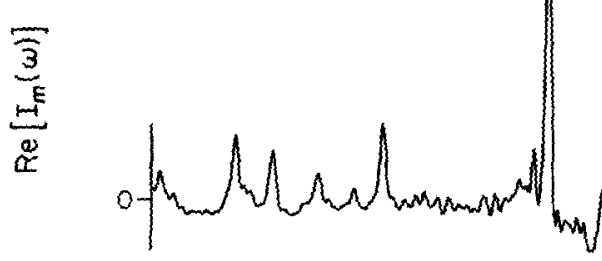

c)

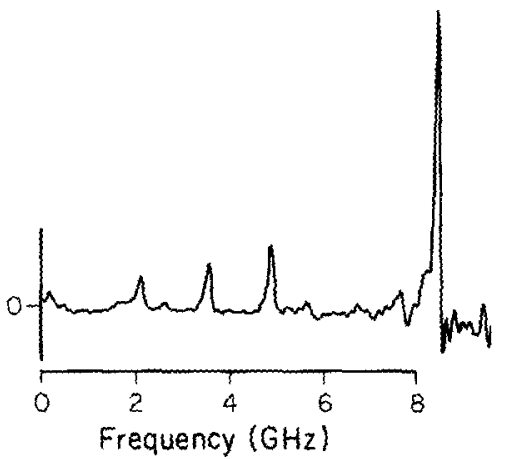

FIG. 4. Fourier spectra of decay residuals (see Sec. II B) for the 390 $\mathrm{cm}^{-3}$ band in the $E_{v i b}=1380 \mathrm{~cm}^{-3}$ spectrum of anthracene for three slightly different excitation energies. All other experimental conditions were the same for the decays. The top spectrum corresponds to excitation at the band maximum, the middle spectrum to excitation about $2 \mathrm{~cm}^{-1}$ to the red of this, and the bottom spectrum about $2 \mathrm{~cm}^{-1}$ to the blue of the maximum. Prominent Fourier bands are labeled with their frequencies in $\mathrm{GHz}$. Note that some bands change in relative intensity from spectrum to spectrum. Note also that all bands have positive amplitudes $(+1$ phases).

0.75 for the $3.5,4.9$, and $8.4 \mathrm{GHz}$ components, respectively.

The decays of the bands at $1460,1460+390$, and $1460+1408 \mathrm{~cm}^{-1}$ (group II) are presented in Fig. 5. All three decays look similar, as is confirmed by Fourier analysis. The apparently decreased modulation depths in the lower two decays are attributable to congestion in the spectral regions in which their corresponding fluorescence bands occur. Figure 6 presents the Fourier spectrum which results from transforming the modulated residual of the $1460 \mathrm{~cm}^{-1}$ decay. Only three prominent beat components at $3.5,4.9$, and $8.4 \mathrm{GHz}$ appear. Note that the phases of the 4.9 and $8.4 \mathrm{GHz}$ components are negative, while the $3.5 \mathrm{GHz}$ component has a positive phase. The absolute modulation depths of the three components, in order of increasing frequency, are found from Fourier analysis to be $0.14,-0.63$, and -0.26 .

The decays of the bands at $1429,1528,1528+390$, and $1528+1408 \mathrm{~cm}^{-1}$ (group III) are presented in Fig.

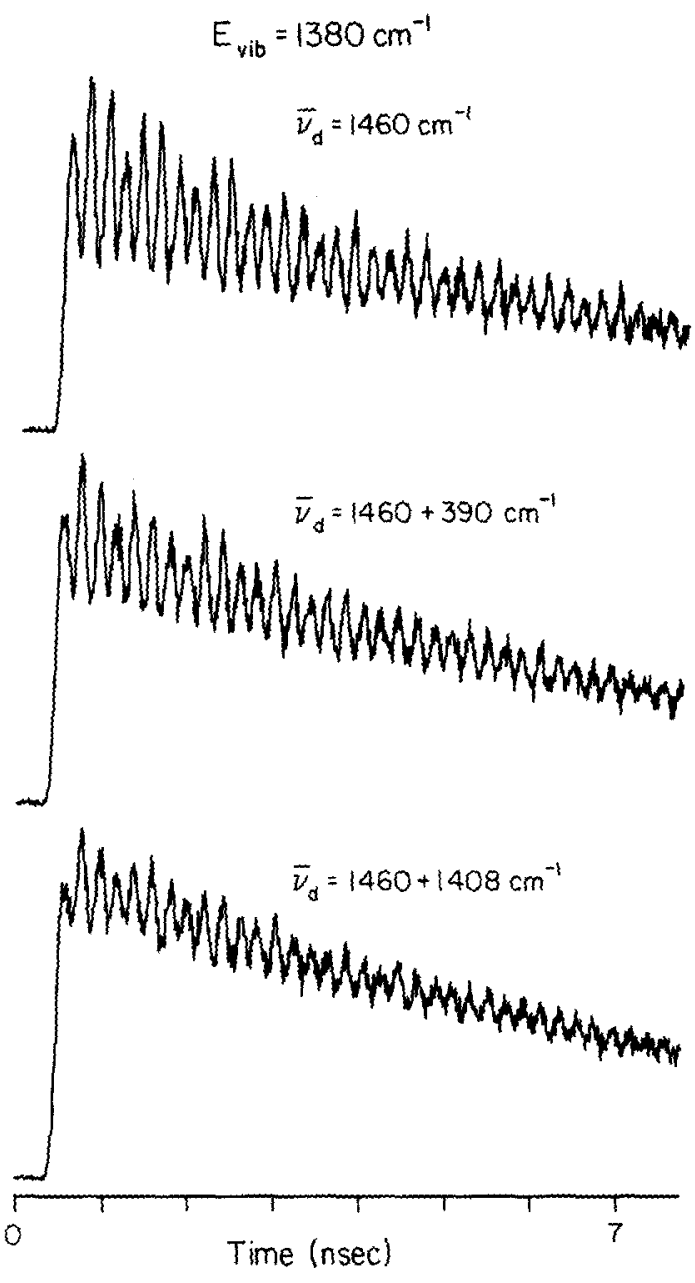

FIG. 5. Fluorescence decays of group II bands in the $E_{\text {vit }}=1380 \mathrm{~cm}^{-1}$ spectrum of anthracene. The shifts of the bands from the excitation energy are given in the figure. From top to bottom $R=0.5,1.0$, and $1.6 \AA$.

7. Note the similar appearance of the decays. The Fourier transform spectrum of the $1528 \mathrm{~cm}^{-1}$ decay is presented in Fig. 8 and is representative of the other Fourier spectra.

$$
\begin{aligned}
E_{v i b} & =1380 \mathrm{~cm}^{-1} \\
\tilde{\nu}_{\sigma} & =1460 \mathrm{~cm}^{-1}
\end{aligned}
$$

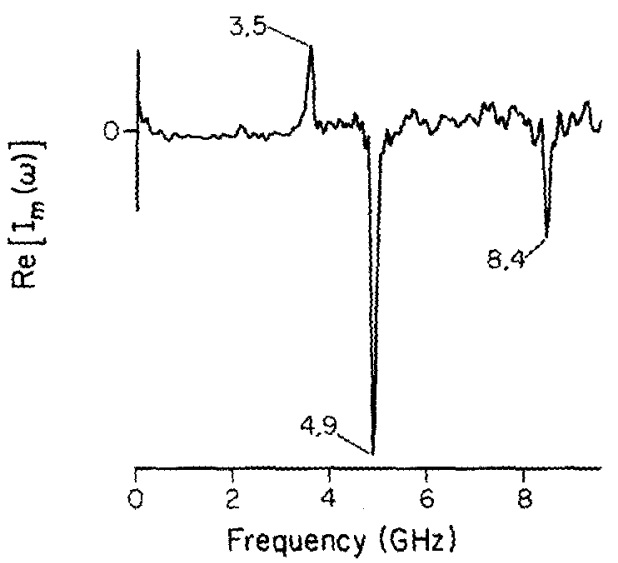

FIG. 6. Fourier spectrum of the residual of the decay of Fig. 5 (top). Fourier bands are labeled with their frequency values in GHz. Note that two of these bands have -1 phases and one has a +1 phase. 


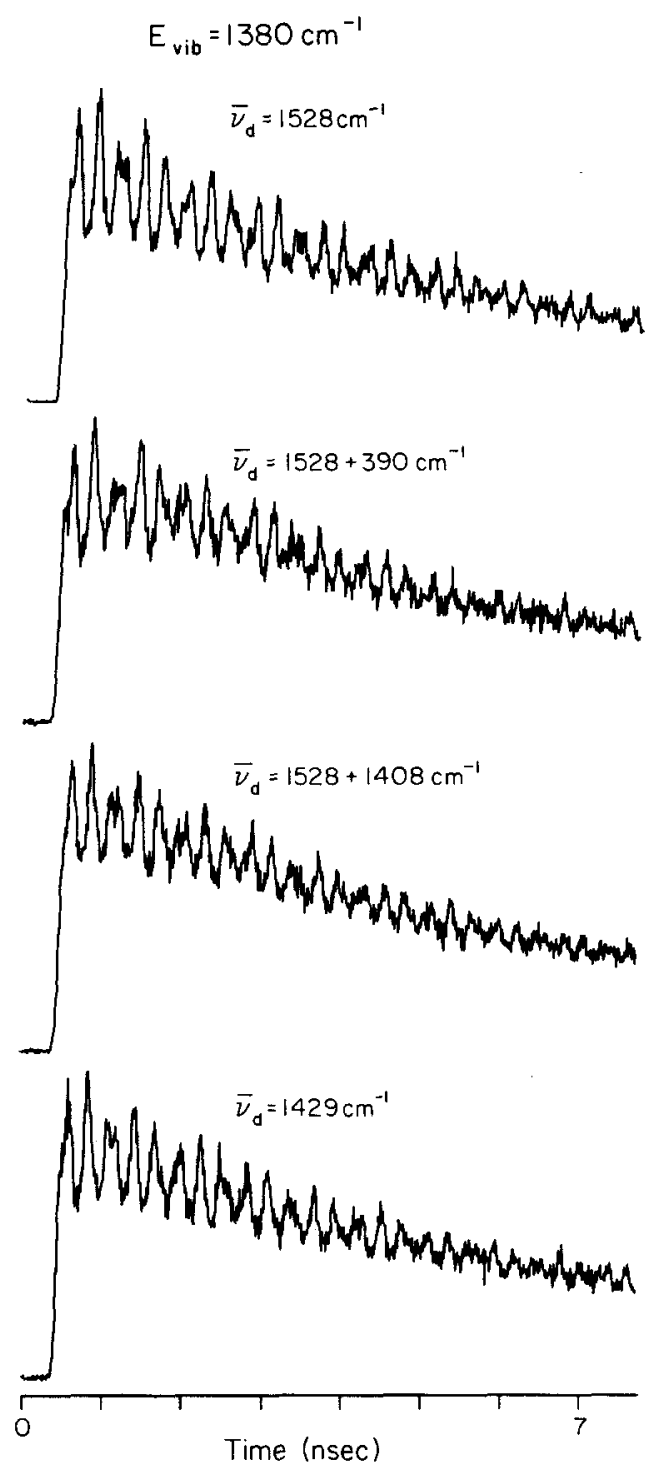

FIG. 7. Fluorescence decays of group III bands in the $E_{\text {vib }}=1380 \mathrm{~cm}^{-1}$ spectrum of anthracene. The shifts of the bands from the excitation energy are given in the figure. From top to bottom $R=0.8,1.0,1.0$, and $1.3 \AA$.

Again, the $3.5,4.9$, and $8.4 \mathrm{GHz}$ components are the only discernible components. Now, however, the phase of the $4.9 \mathrm{GHz}$ component is positive, while the other two components have negative phases. The derived absolute modulation depths for the decay of this band are quite sensitive to detection wavelength and resolution, as is reasonable considering its position in the spectrum (Fig. 2). The values for the absolute modulation depths derived from the decay of the $1528 \mathrm{~cm}^{-1}$ band are -0.45 , 0.16 , and -0.41 , for the three components in order of increasing frequency.

The fourth group of bands which shall be considered (group IV) are the three which occur in the congested $1480-1520 \mathrm{~cm}^{-1}$ region of the spectrum. In this region the ability to spectrally isolate the various bands to measure a decay, while still maintaining a reasonable signal level, was limited. Nevertheless, modulated decays were observed, as shown in Fig. 9. For some of these decays, Fourier analysis (not shown) reveals some contri-

$$
\begin{aligned}
E_{\text {vib }} & =1380 \mathrm{~cm}^{-1} \\
\bar{v}_{d} & =1528 \mathrm{~cm}^{-1}
\end{aligned}
$$

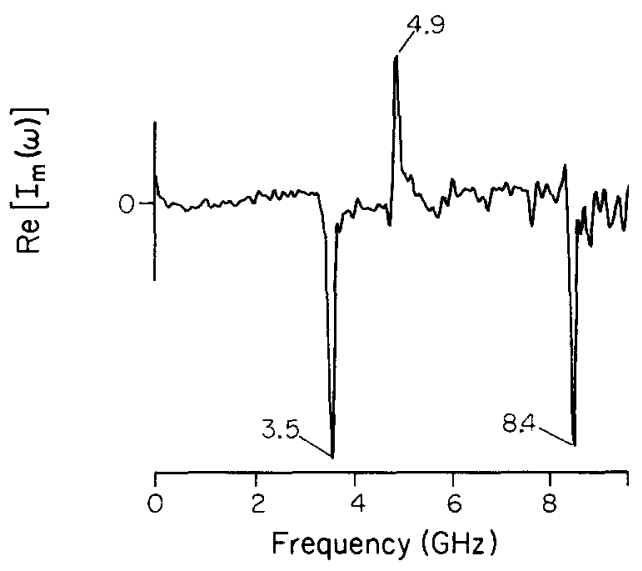

FIG. 8. Fourier spectrum of the residual of the decay of Fig. 7 (top). Bands are labeled with their frequency values in $\mathrm{GHz}$. Note that two of the bands have -1 phases and one has a +1 phase. Note also, however, that the phase behavior is different from that in Fig. 6.

bution from the $3.5,4.9,8.4 \mathrm{GHz}$ triplet of beat components. Also present, however, unlike for the decays of groups II and III, are some of the other beat components

$$
E_{\text {vib }}=1380 \mathrm{~cm}^{-1}
$$
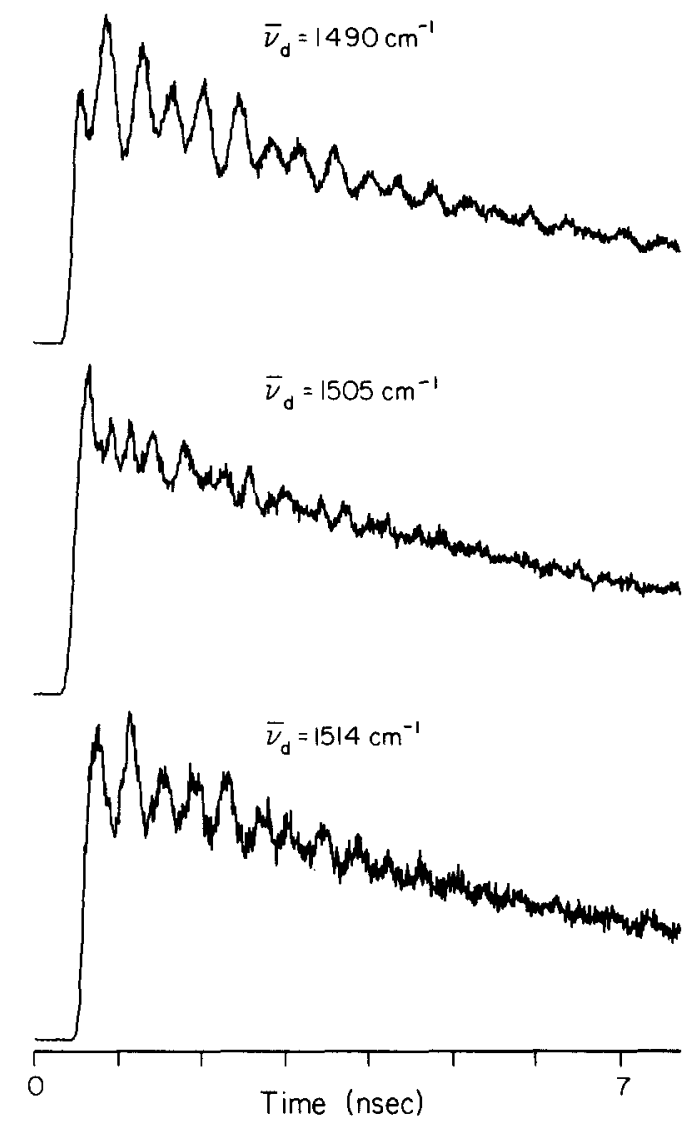

FIG. 9. Fluorescence decays of group IV bands in the $E_{\text {vib }}=1380 \mathrm{~cm}^{-1}$ spectrum of anthracene. The shifts of the bands from the excitation energy are given in the figure. For all decays $R=1.6 \AA$. 
that appear in the group I decays. In particular, all or some of the components at $1.8,2.6$, and $4.3 \mathrm{GHz}$ appear prominently in these decays. Moreover, they exhibit phase behaviors that are different from that of the 390 $\mathrm{cm}^{-1}$ decay: the $1490 \mathrm{~cm}^{-1}$ decay has the 1.8 and 4.3 $\mathrm{GHz}$ components with negative phases and the $2.6 \mathrm{GHz}$ component with positive, while the $1505 \mathrm{~cm}^{-1}$ decay has the $4.3 \mathrm{GHz}$ component with positive phase and the other two with negative phases.

Finally, we consider the decay of the band at 1125 $\mathrm{cm}^{-1}$, which has been extensively studied previously ${ }^{1,2}$ using $\sim 400 \mathrm{ps}$ temporal resolution. The decay of this band using 80 ps temporal resolution is presented in Fig. 10. The decay was taken under expansion conditions ${ }^{2}$ which favor the appearance of the $0.2 \mathrm{GHz}$ beat component (a $0.5 \mathrm{GHz}$ component predominates under less efficient rotational cooling conditions ${ }^{2}$ ). This component is clearly present in the decay. Moreover, Fourier analysis confirms our earlier assertion ${ }^{2}$ that the component has a negative phase. While higher frequency components also appear to modulate the $1125 \mathrm{~cm}^{-1}$ decay, the difficulty in obtaining relatively noiseless data for this weak fluorescence band has precluded any detailed consideration of these components.

\section{Interpretation}

As we have stated above, the characteristics of the dispersed fluorescence spectrum and the spectral dependence of the fluorescence decays corresponding to excitation at $E_{\mathrm{vib}}=1380 \mathrm{~cm}^{-1}$ in anthracene both indicate that the experimental results are the dynamical manifes-
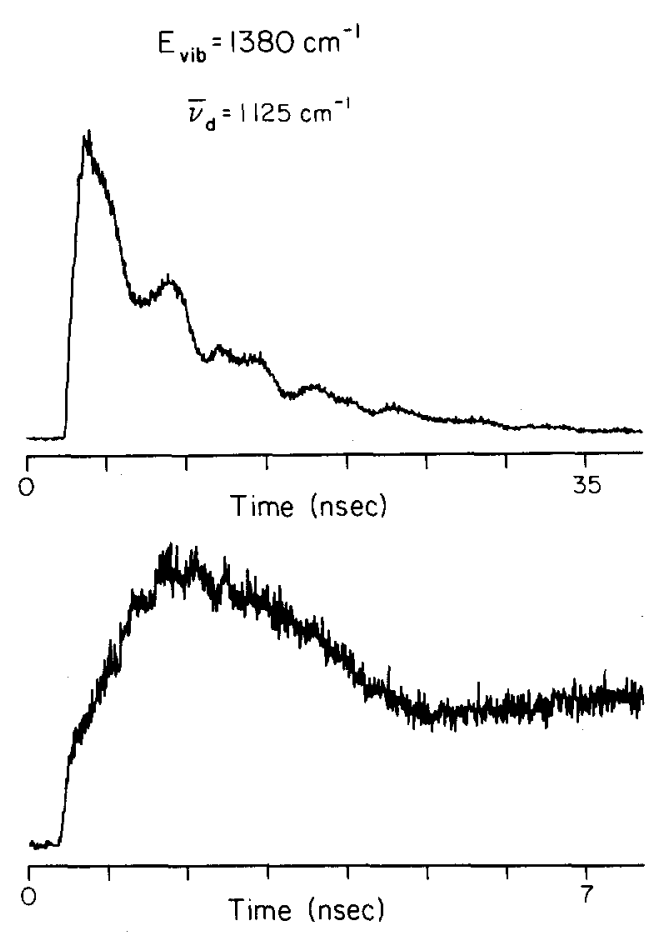

FIG. 10. Fluorescence decays of the $1125 \mathrm{~cm}^{-1}$ band in the $E_{\text {vib }}=1380$ $\mathrm{cm}^{-1}$ spectrum of anthracene on long (top) and short (bottom) time scales. The decays are modulated principally at $0.2 \mathrm{GHz}$. The top decay was taken with $R=1.6 \AA$ and the bottom with $R=3.2 \AA$. tations of vibrational coupling in the $S_{1}$ state. One would, however, like to characterize this coupling more fully. The major problem is to determine the number of levels involved. According to I this can be done if the number of beat components and the number of beat triplets are known. (By a beat triplet ${ }^{7}$ we mean the three beat components $\omega_{I J}, \omega_{J K}$, and $\omega_{I K}$. Note that two of the frequencies of such a triplet sum to the third.) The number of components (8) and the number of triplets (2) evident from the $390 \mathrm{~cm}^{-1}$ decay do not correspond to the theoretical expectations for any number of coupled levels. This could be due to at least two causes, as pointed out in Secs. IV B and IV D of paper I. Either some beat components are too weak or of too high a frequency to be observed, or the finite excitation bandwidth spans at least two independent sets of coupled levels. Here, there is strong reason to believe that the latter situation is the one that obtains. This conclusion arises from the fact that the relative Fourier amplitudes of the various beat components are dependent on excitation energy; the triplet of frequencies at $1.8,2.6$, and $4.3 \mathrm{GHz}$ is enhanced relative to the triplet at $3.5,4.9$, and $8.4 \mathrm{GHz}$ when the excitation is detuned to the red and vice versa when the excitation is detuned to the blue (Fig. 4). Moreover, the other groups of bands in the dispersed fluorescence spectrum have decays in which one or the other beat triplet dominates. This is consistent with the manifestations of two sets of three coupled levels, the energy separation of the two sets being small enough to be within the excitation bandwidth, but large enough to preclude the observation of any beat frequencies corresponding to energy differences between two states belonging to two different sets. ${ }^{17}$ In the nomenclature of Sec. IV D (case 4) of I, the bands of group I would correspond to overlapping $a$ - and $a^{\prime}$-type bands. The bands of groups II and III would then correspond to $b$ - and $c$-type bands, respectively, since the phase behaviors of the two types of decays are consistent with theoretical expectations for the two non- $a$-type bands of a three-level system: two -1 phases and one +1 phase for each decay. The 1490 and $1505 \mathrm{~cm}^{-1}$ bands would be assigned as $b^{\prime}$ - and $c^{\prime}$-type bands. For these too, the beat phase behaviors of the decays are consistent with the expectations of theory for the two non- $a$-type bands of a three-level system.

The sums of the modulation depths for the non- $a$ type bands, by Eq. (2.17) of the previous paper, should be equal to -1 . However, the $b$-type $1460 \mathrm{~cm}^{-1}$ decay gives a value of -0.75 , and the $c$-type $1528 \mathrm{~cm}^{-1}$ decay gives a value of -0.70 . As was pointed out in Sec. II B, this could be due to a number of sources of experimental error, particularly problems with spectral resolution and with response function drifts. Alternatively, this could be due to unobserved eigenstates (see Secs. IV A and IV B of I). For example, the $|a\rangle,|b\rangle$, and $|c\rangle$ zero-order levels may be just three of four coupled levels, with the coupling being such that three of the eigenstates are closely spaced, with the fourth being widely separated from these three. It is likely that both types of influences play some role in the less than ideal values derived from experiment. In any case, the values -0.75 and -0.70 are close enough 
to -1 to warrant tentative treatment of the unprimed system in terms of three coupled levels.

One may also note that two of the beat frequencies modulating the $390 \mathrm{~cm}^{-1}$ band remain unexplained by the scheme, presented above, of two sets of three coupled levels; the components at 0.2 and $2.1 \mathrm{GHz}$. We have previously shown ${ }^{2}$ that for the $E_{\text {vib }}=1380 \mathrm{~cm}^{-1}$ excitation there exists a marked rotational level dependence to the coupling which gives rise to the $0.2 \mathrm{GHz}$ component; the coupling only occurring significantly for certain higher energy rotational levels. This combines with the facts that (1) the $0.2 \mathrm{GHz}$ modulation of the $390 \mathrm{~cm}^{-1}$ band is relatively weak and (2) any other bands exhibiting decays with significant modulation at this frequency (e.g., the $1125 \mathrm{~cm}^{-1}$ band) appear only with weak intensity in the fluorescence spectrum, to indicate that the $0.2 \mathrm{GHz}$ component is a manifestation of a coupling that figures only in a minor way, and at higher rotational energy levels, in the IVR dynamics at this excess vibrational energy. Whether or not this coupling is associated with the $|a\rangle,|b\rangle$, and $|c\rangle$ states, the $\left|a^{\prime}\right\rangle,\left|b^{\prime}\right\rangle$, and $\left|c^{\prime}\right\rangle$ states, or with neither, cannot be answered without higher resolution excitation contour experiments. As for the beat component at $2.1 \mathrm{GHz}$, one is handicapped by the fact that no bands have been observed, other than those of group I, which exhibit in their decays a prominent component at this frequency. This suggests that the component does not arise from coupling involving the unprimed or primed set of states. It is possible that it does arise from the same coupling that gives rise to the $0.2 \mathrm{GHz}$ component or that it represents a manifestation of two more coupled levels spanned by the bandwidth of the excitation pulse.

\section{Calculation of $H_{0}$}

As we have shown in Sec. III of the preceding paper, ${ }^{7}$ it is possible to use observed quantum beat parameters to calculate the vibrational Hamiltonian matrix in the zero-order basis set (i.e., $H_{0}$ ). In this section we do this for the three-level system composed of the $|a\rangle,|b\rangle$, and $|c\rangle$ coupled levels which give rise to the fluorescence decays modulated at $3.5,4.9$, and $8.4 \mathrm{GHz}$. Our choice of this coupled system is based on the quality of the data associated with it and the fact that these levels contribute most prominently to the fluorescence arising from the $1380 \mathrm{~cm}^{-1}$ excitation band.

We start by labeling the beat frequencies such that $\left|\omega_{12} / 2 \pi\right|=3.5,\left|\omega_{23} / 2 \pi\right|=4.9$, and $\left|\omega_{13} / 2 \pi\right|=8.4 \mathrm{GHz}$. In so doing, the following is obtained for the diagonal Hamiltonian matrix:

$$
E=E_{0} I \pm\left(\begin{array}{lll}
0.0 & 0.0 & 0.0 \\
0.0 & 3.5 & 0.0 \\
0.0 & 0.0 & 8.4
\end{array}\right)
$$

where $E_{0}$ is an unknown constant, and where the energy values are expressed in $\mathrm{GHz}$.

Next, we calculate the $\alpha_{I a}$ from the relative modulation depths of the $390 \mathrm{~cm}^{-1}$ decay. Using

$$
\frac{M_{a}\left(\omega_{23}\right)}{M_{a}\left(\omega_{13}\right)}=0.22 \text { and } \frac{M_{a}\left(\omega_{23}\right)}{M_{a}\left(\omega_{12}\right)}=1.31,
$$

Eqs. (3.3a) and (3.3b) of paper I, and the normalization condition, one obtains

$$
\alpha_{1 a}=0.629 ; \quad \alpha_{2 a}=0.295 ; \quad \alpha_{3 a}=0.720 ;
$$

where we have exercised the freedom to choose all these values to be positive. ${ }^{7}$

Next, we may calculate either the $\alpha_{I b}$ or the $\alpha_{I c}$ values. We choose the $\alpha_{1 b}$ because the $1460 \mathrm{~cm}^{-1} b$-type band is very strong and relatively isolated. Therefore, one expects that the values for the relative modulation depths for this band are fairly accurate. Using

$$
\frac{M_{b}\left(\omega_{23}\right)}{M_{b}\left(\omega_{13}\right)}=2.47 \text { and } \frac{M_{b}\left(\omega_{23}\right)}{M_{b}\left(\omega_{12}\right)}=-4.42,
$$

Eqs. (3.4a) and (3.4b) of paper $I$, and the normalization condition for the $\alpha_{l b}$ one obtains

$$
\alpha_{1 b}=0.152 ; \quad \alpha_{2 b}=0.799 ; \quad \alpha_{3 b}=-0.582 ;
$$

where our choice of $\alpha_{1 b}$ as positive fixes the signs of $\alpha_{2 b}$ and $\alpha_{3 b}$.

Finally, we calculate the $\left|\alpha_{I c}\right|$ using the relations

$$
\sum_{\gamma=a, b, \cdots} \alpha_{I_{\gamma}}^{2}=1 \text { for } I=1,2,3,
$$

and then determine the signs of the coefficients using the quantum beat phase behavior of the c-type bands (see Fig. 8). This yields

$$
\alpha_{1 c}=-0.762 ; \quad \alpha_{2 c}=0.525 ; \quad \alpha_{3 c}=0.378 \text {. }
$$

As a whole, the eigenvector coefficient matrix is

$$
C=\left(\begin{array}{rrr}
0.629 & 0.295 & 0.720 \\
0.152 & 0.799 & -0.528 \\
-0.762 & 0.525 & 0.378
\end{array}\right),
$$

with the columns labeled by the molecular eigenstates and the rows labeled by the zero-order states. As pointed out in paper $I$, a number of tests can be made on this calculated matrix. Firstly, the orthogonality of its rows and columns may be checked. Calculating the six possible cross products, one finds no deviations from orthogonality of over $5.3^{\circ}$ and an average deviation of about $4^{\circ}$. Secondly, the absolute modulation depths can be calculated from $C$ and compared with experimental values. Such a comparison appears in Table I. One sees that the calculated magnitudes are generally $\sim 20 \%-30 \%$ higher than the experimental ones, probably due to experimental limitations or unobserved eigenstates.

Using the values for $C$ and $E$, one can calculate $H_{0}$ up to various constants by Eq. (3.1) of I. Doing so, one obtains

$$
H_{0}^{1380}=E_{0} I \pm\left(\begin{array}{rrr}
4.66 & -2.70 & 2.83 \\
-2.70 & 5.08 & -0.38 \\
2.83 & -0.38 & 2.16
\end{array}\right),
$$

where the three rows and columns are both labeled by $a$, $b$, and $c$. Note that the relative signs of the off-diagonal 
TABLE I. Calculated and observed absolute modulation depths for bands in the $E_{\text {vib }}=1380 \mathrm{~cm}^{-1}$ spectrum of anthracene.

\begin{tabular}{lcccc}
\hline \hline Band type & $\begin{array}{c}3.5 \mathrm{GHz} \\
\text { beat }\end{array}$ & $\begin{array}{c}4.9 \mathrm{GHz} \\
\text { beat }\end{array}$ & $\begin{array}{c}8.4 \mathrm{GHz} \\
\text { beat }\end{array}$ & $\begin{array}{c}\text { Sum of } \\
\text { modulations }\end{array}$ \\
\hline $\begin{array}{l}a \text { type: } \\
\text { calculated }\end{array}$ & +0.16 & +0.21 & +0.95 & +1.26 \\
observed & +0.12 & +0.16 & +0.75 & +1.03 \\
$\begin{array}{l}b \text { type: } \\
\quad \text { calculated } \\
\text { observed }\end{array}$ & +0.22 & -0.86 & -0.35 & -0.99 \\
$c$ type: & +0.14 & -0.63 & -0.26 & -0.75 \\
$\quad$ calculated & -0.45 & +0.26 & -0.80 & -0.99 \\
observed & -0.45 & +0.16 & -0.41 & -0.70 \\
\hline \hline
\end{tabular}

elements are not unique. ${ }^{7}$ This is because the rows of $C$ are only fixed up to an overall sign by the quantum beat results. Thus, instead of the distribution $(-1,+1,-1)$ for the signs of the interaction matrix elements $\left(V_{a b}, V_{a c}\right.$, $\left.V_{b c}\right)$ one also could have obtained $(+1,-1,-1),(+1,+1$, $+1)$, or $(-1,-1,+1)$ by different choices for the overall signs of the rows of $C$.

\section{B. $E_{\text {vib }}=1420 \mathrm{~cm}^{-1}$ : The $a_{g}(5)$ mode}

The $1420 \mathrm{~cm}^{-1}$ excitation band, which has been assigned in Ref. 14, is the one for which phase-shifted quantum beats were first discovered. ${ }^{3}$ At that time the beating behavior appeared to arise from a coupled twolevel system. Since then, with better time resolution it has become evident that the vibrational dynamics at this energy involve at least three coupled levels. ${ }^{4}$ In this section we present and analyze the results for this band in a manner similar to the treatment of the $1380 \mathrm{~cm}^{-1}$ band in the previous section.

\section{Results}

The dispersed fluorescence spectrum resulting from the excitation of the $1420 \mathrm{~cm}^{-1}$ band is shown in Fig. 11 together with a high resolution spectrum of the region near the $S_{1}$ origin. The general characteristics of the spectrum are quite similar to the $1380 \mathrm{~cm}^{-1}$ spectrum; i.e., there is a mix of assignable and unassignable bands, there is some resonance fluorescence, and there is resolvable spectral congestion in and to the red of the $S_{1}$ origin region.

Also similar to the $1380 \mathrm{~cm}^{-1}$ results, the timeresolved results reveal fluorescence decays which are beatmodulated and which depend on detection wavelength. Again, we shall discuss the various types of decays separately.

Figure 12 (top) presents the decay of the $390 \mathrm{~cm}^{-1}$ band. Fourier analysis of the decay [Fig. 13 (top)] reveals three prominent beat components at 1.0, 9.7, and 10.7 $\mathrm{GHz}$, all with positive phases and with absolute modulation depths of $0.55,0.36$, and 0.52 , respectively. The 390 $\mathrm{cm}^{-1}$ decay is representative of a number of other decays, as well; those of the 0,780 , and $1168 \mathrm{~cm}^{-1}$ bands. (Note that all of these bands have counterparts in the group I, $a$-type bands of the $1380 \mathrm{~cm}^{-1}$ spectrum and that they

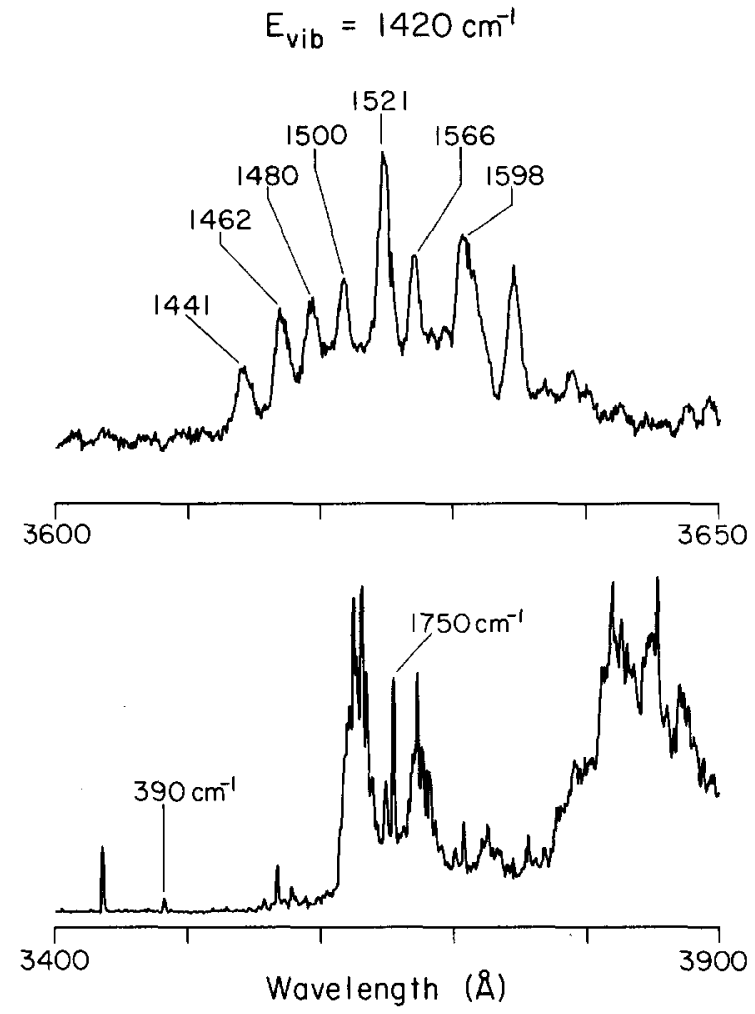

FIG. 11. Dispersed fluorescence resulting from excitation of jet-cooled anthracene to $S_{1}+1420 \mathrm{~cm}^{-1}$. The upper portion was taken with $R$ $=0.6 \AA$ and the lower with $R=1.6 \AA$. Various bands in the spectra are marked with their shifts in $\mathrm{cm}^{-1}$ from the excitation energy.
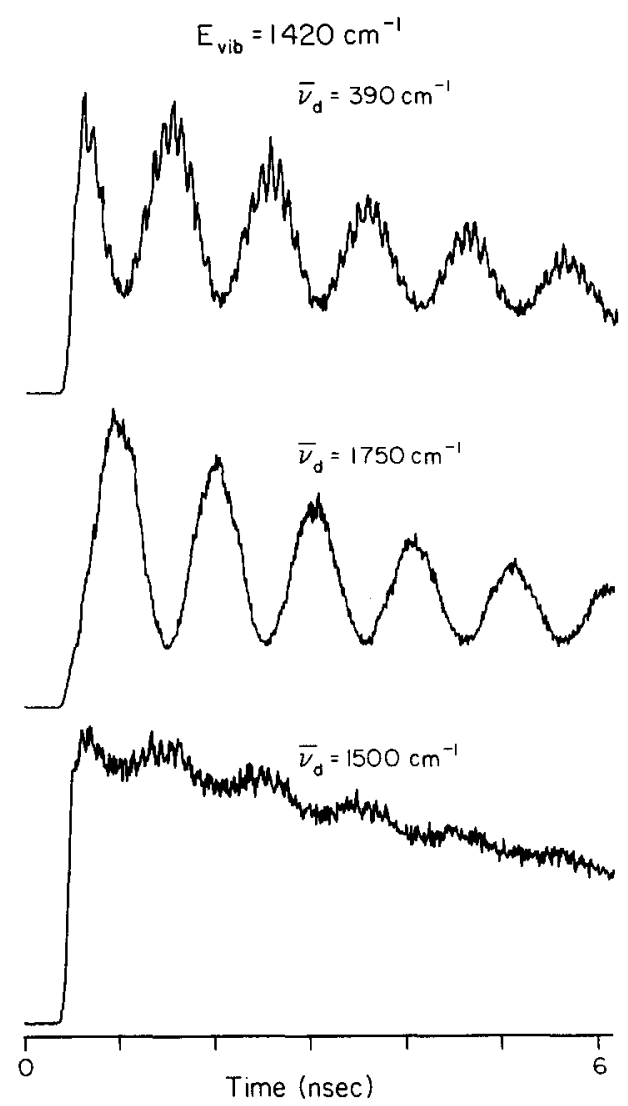

FIG. 12. Representative decay types for fluorescence bands in the $E_{\text {vib }}$ $=1420 \mathrm{~cm}^{-1}$ spectrum of anthracene. The wave number shifts of the bands from the excitation energy are given in the figure. From top to bottom $R=16.0,1.6$, and $1.6 \AA$ 

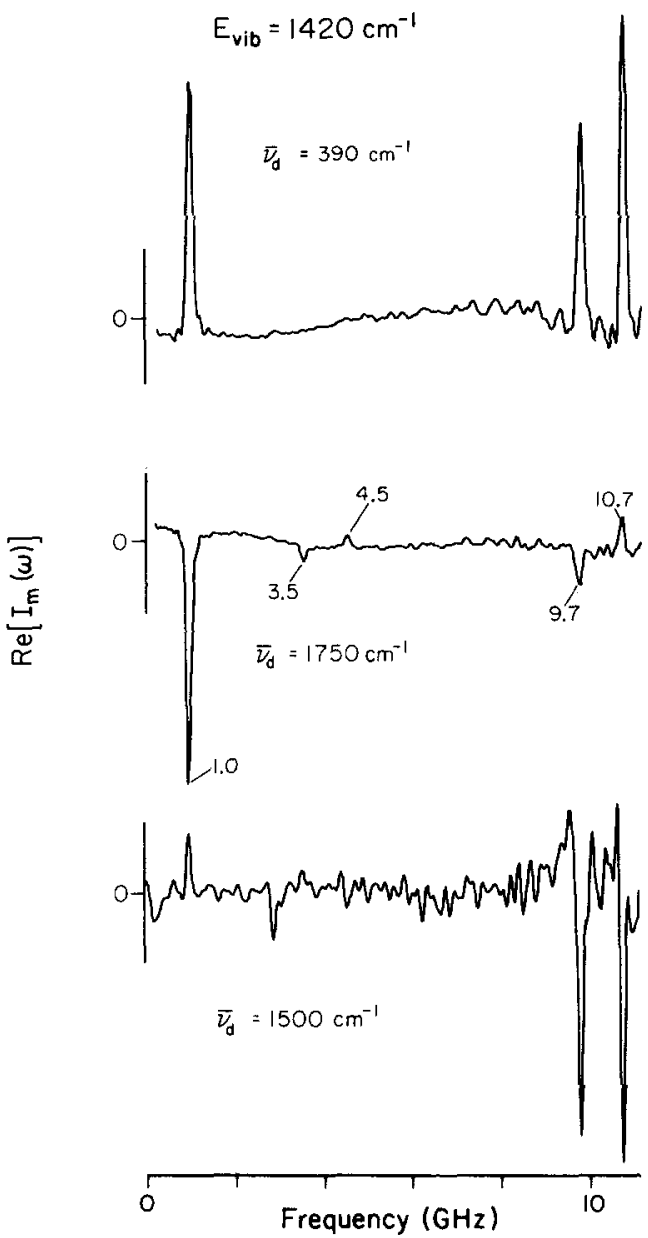

FIG. 13. Fourier spectra of the residuals of the decays of Fig. 12. Bands in the middle spectrum are labeled with values in $\mathrm{GHz}$. Although there appear to be more than three components in the lower spectrum, only the ones at $1.0,9.7$, and $10.7 \mathrm{GHz}$ are reproducible. Any other apparent bands in this spectrum are therefore probably noise.

are all assignable in terms of intervals associated with optically active modes. ${ }^{14}$ ) There are also other bands in the congested region of the spectrum near the $S_{1}$ origin which appear to exhibit decay behavior similar to the 390 $\mathrm{cm}^{-1}$ band (e.g., the $1480 \mathrm{~cm}^{-1}$ band).

Figure 12 (middle) presents the decay of the 1750 $\mathrm{cm}^{-1}$ band in the dispersed fluorescence. As we have reported, ${ }^{3,4}$ this decay shows an obvious $180^{\circ}$ phase shift of the $1.0 \mathrm{GHz}$ beat component relative to the +1 phase of the same beat component in the $390 \mathrm{~cm}^{-1}$ decay. Fourier analysis [Fig. 13 (middle)] reveals that besides this beat component, the decay is also modulated by at least four others: the 9.7 and $10.7 \mathrm{GHz}$ components found in the $390 \mathrm{~cm}^{-1}$ decay, and two more components at 3.5 and $4.5 \mathrm{GHz}$. The absolute modulation depths for these components in order of increasing frequency are $-0.74,-0.06,0.04,-0.11$, and 0.08 . The sum of the modulation depths is -0.79 . Although we have not measured the decays of the bands at $1750+390$ and $1750+1408 \mathrm{~cm}^{-1}$ with 80 ps temporal resolution, previous measurements ${ }^{3}$ of the decays showed that the decay behavior of these bands is consistent with that of the $1750 \mathrm{~cm}^{-1}$ band (i.e., the $1 \mathrm{GHz}$ component was found to have a large modulation depth and a negative phase).

A third band at $1500 \mathrm{~cm}^{-1}$ exhibits different decay behavior than both the 390 and $1750 \mathrm{~cm}^{-1}$ bands. Figure 12 (bottom) presents the decay of this band and Fig. 13 (bottom) the Fourier spectrum of the residual. Despite the noise in the Fourier spectrum, three Fourier components are evident at $1.0,9.7$, and $10.7 \mathrm{GHz}$ with phases of $+1,-1$, and -1 , respectively. Nominal values for the relative modulation depths derived from the $1500 \mathrm{~cm}^{-1}$ decay are $0.2,-0.9$, and -1.0 , in order of increasing beat frequency.

Finally, in Fig. 14 the decays for many of the prominent bands that appear in the region of the $S_{1}$ origin are presented. We shall not extensively analyze these decays here, being that the limitations of spectral and temporal resolution prohibit any very quantitative analysis. It is evident that all of the decays are modulated

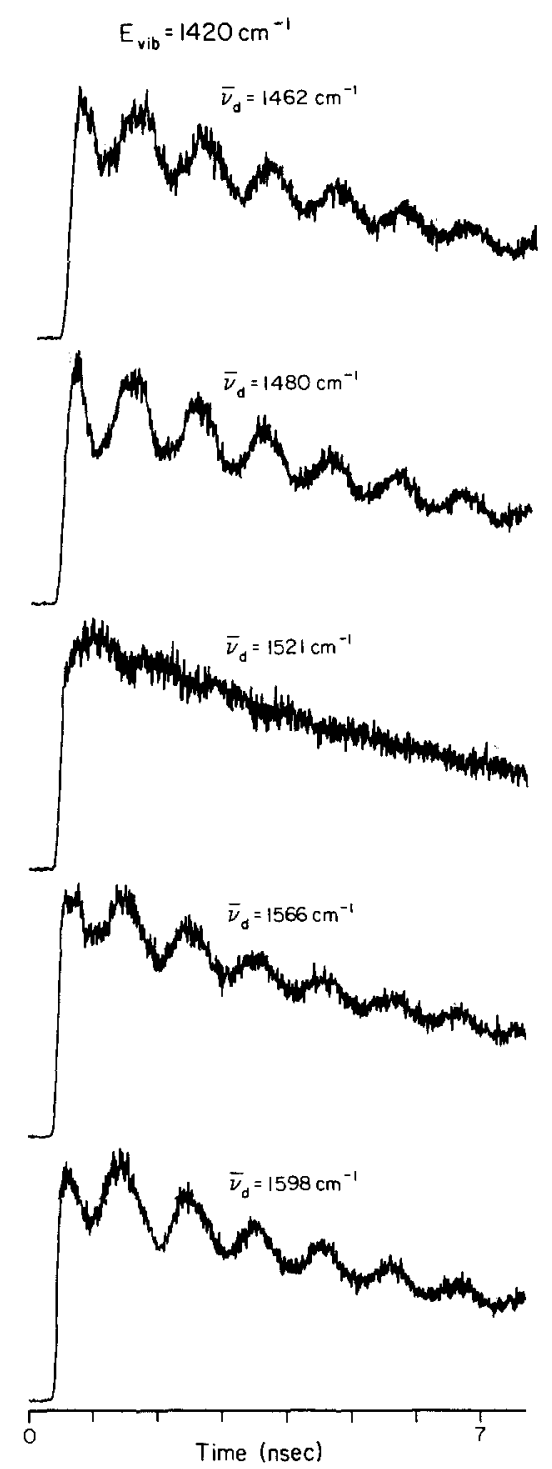

FIG. 14. Fluorescence decays of bands in the $E_{\mathrm{vib}}=1420 \mathrm{~cm}^{-1}$ spectrum of anthracene that occur near the $0_{0}^{\circ}$ transition energy of the molecule [see the spectrum of Fig. 11 (top)]. The wave number shifts of the bands from the excitation energy are given in the figure. From top to bottom $R=1.6,1.6,0.8,1.6$, and $1.6 \AA$ 
to some extent by the $1 \mathrm{GHz}$ beat component. Moreover, some, and perhaps all, are modulated prominently with the 9.7 and $10.7 \mathrm{GHz}$ components, as well.

\section{Interpretation}

As with the results for the $1380 \mathrm{~cm}^{-1}$ band, it is desirable to come to an understanding of the number of coupled levels being manifested in the results for the $1420 \mathrm{~cm}^{-1}$ band. Just considering the Fourier spectrum (Fig. 13) for the $390 \mathrm{~cm}^{-1}$ decay, which exhibits one triplet of beat components, one would come to the conclusion that only three levels were involved. However, the $1750 \mathrm{~cm}^{-1}$ decay, with its five beat components (Fig. 13) is not consistent with this. Instead, the latter decay indicates that the $1.0,9.7$, and $10.7 \mathrm{GHz}$ beat frequencies are just three of the six energy splittings expected for a four level system. Considering the five beat components of the $1750 \mathrm{~cm}^{-1}$ decay as whole, one sees that they are consistent with the manifestations of a four-level system, if it is assumed that one beat component, at either 6.2 or 14.2 $\mathrm{GHz}$, is undetectable due to weak modulation or (in the case of the $14.2 \mathrm{GHz}$ component) an unresolvable frequency. In both cases there would be six beat components and four beat triplets, as expected ${ }^{7}$ for a four-level system. [The former case would have the triplets (1.0, $3.5,4.5)$, $(1.0,9.7,10.7),(3.5,6.2,9.7)$ and $(4.5,6.2$, 10.7), whereas the latter would have the triplets $(1.0,3.5$, $4.5),(1.0,9.7,10.7),(3.5,10.7,14.2)$, and $(4.5,9.7$, 14.2).] Note that no other values for the sixth beat frequency would be consistent with a four-level system. Note also how the $1420 \mathrm{~cm}^{-1}$ case differs from the 1380 $\mathrm{cm}^{-1}$ case, for which six beat frequencies were assigned to two independent three-level systems: unlike in the $1420 \mathrm{~cm}^{-1}$ case, in the $1380 \mathrm{~cm}^{-1}$ case the beat frequencies are such that there is no way to form four triplets.

All in all, the results indicate that the $390 \mathrm{~cm}^{-1}$ band is an $a$-type band and the $1750 \mathrm{~cm}^{-1}$ band is a non- $a$ type band (say $b$ type) of a coupled four-level system. The existence of a situation wherein four vibrational levels are coupled implies the existence of four types of fluorescence bands, each with different quantum beat phase behaviors. Obviously, we have observed two of these band types. We can also identify a third one: the band at $1500 \mathrm{~cm}^{-1}$, which we call $c$ type. Our inability to identify a $d$-type band is probably due to problems associated with the spectral overlap of fluorescence bands.

\section{Calculation of $\mathrm{H}_{0}$}

To calculate a four-by-four $H_{0}$ matrix for $E_{\text {vib }}$ $=1420 \mathrm{~cm}^{-1}$, an initial assumption must be made involving the value for the unknown sixth beat frequency. We shall choose the lower value $6.2 \mathrm{GHz}$, since using it seems to give values for the elements of $C$ which more nearly approximate those of an orthonormal matrix than the values calculated using $14.2 \mathrm{GHz}$ for the sixth beat frequency. Hence we label the beat frequencies such that $\left|\omega_{12} / 2 \pi\right|=1.0 \mathrm{GHz},\left|\omega_{23} / 2 \pi\right|=3.5 \mathrm{GHz},\left|\omega_{34} / 2 \pi\right|=6.2$
$\mathrm{GHz},\left|\omega_{13} / 2 \pi\right|=4.5 \mathrm{GHz},\left|\omega_{24} / 2 \pi\right|=9.7 \mathrm{GHz}$, and $\left|\omega_{14}\right|$ $2 \pi \mid=10.7 \mathrm{GHz}$. This gives an eigenvalue matrix

$$
E=E_{0} I \pm\left(\begin{array}{rrrr}
0.0 & 0.0 & 0.0 & 0.0 \\
0.0 & 1.0 & 0.0 & 0.0 \\
0.0 & 0.0 & 4.5 & 0.0 \\
0.0 & 0.0 & 0.0 & 10.7
\end{array}\right)
$$

Next the eigenvector coefficient matrix $C$ must be considered. We start with the calculation of the $\alpha_{I a}$ from the relative modulation depths of the three detectable Fourier components modulating the $390 \mathrm{~cm}^{-1} a$-type band. These values give

$$
\alpha_{2 a}^{2}=0.600 \alpha_{1 a}^{2} \text { and } \alpha_{4 a}^{2}=0.609 \alpha_{1 a}^{2} .
$$

Because the Fourier amplitudes of the $\omega_{13}, \omega_{23}$, and $\omega_{34}$ components are so weak as to be undetectable, there is no way to calculate $\alpha_{3 a}$. One only knows that the value must be small. Thus, we make an assumption and fix the value of $\alpha_{3 a}$ at 0.05 . Then, with the normalization condition one finds the following values for the $\alpha_{I a}$ :

$$
\alpha_{1 a}=0.672, \quad \alpha_{2 a}=0.521, \quad \alpha_{3 a}=0.050, \quad \alpha_{4 a}=0.525 \text {. }
$$

The next step is to calculate the $\alpha_{I b}$. Using the relative modulation depths of the beat components of the $1750 \mathrm{~cm}^{-1} b$-type band and the normalization condition on the $\alpha_{I b}$ one obtains

$$
\begin{array}{ll}
\alpha_{1 b}=-0.503, & \alpha_{2 b}=0.699, \\
\alpha_{3 b}=-0.498, & \alpha_{4 b}=-0.095 .
\end{array}
$$

The $\alpha_{I c}$ could conceivably be calculated using the relative modulation depths of the $1500 \mathrm{~cm}^{-1}, c$-type band, but for the fact that some of the beat components are lost in the noise of the pertinent Fourier spectrum. Nevertheless, one can use

$$
\frac{M_{c}\left(\omega_{14}\right) \alpha_{2 a}}{M_{c}\left(\omega_{24}\right) \alpha_{1 a}} \alpha_{2 c}=\alpha_{1 c}
$$

to obtain $\alpha_{1 c}=0.86 \alpha_{2 c}$. Then using the orthogonality of the $\alpha_{I c}$ with the $\alpha_{I b}$ and the $\alpha_{I a}$, and, finally, the normalization of the $\alpha_{I c}$, one obtains

$$
\alpha_{1 c}=0.316, \alpha_{2 c}=0.367, \alpha_{3 c}=0.350, \alpha_{4 c}=-0.802 \text {. }
$$

Lastly, the $\left|\alpha_{I d}\right|$ can be calculated using the relations

$$
\sum_{\gamma=a, b, \cdots} \alpha_{I \gamma}^{2}=1 \text { for } I=1,4 \text {. }
$$

The relative signs of the $\alpha_{I d}$ can then be obtained from orthogonality requirements.

The calculated eigenvector matrix is

$$
C=\left(\begin{array}{rrrr}
0.672 & 0.521 & 0.050 & 0.525 \\
-0.503 & 0.699 & -0.498 & -0.095 \\
0.316 & 0.367 & 0.350 & -0.802 \\
0.442 & -0.324 & -0.792 & -0.269
\end{array}\right)
$$

The largest deviation from orthogonality for any of the rows or columns is $3.0^{\circ}$, with the average being about 
TABLE II. Calculated and observed absolute modulation depths for bands in the $E_{\mathrm{vib}}=1420 \mathrm{~cm}^{-1}$ spectrum of anthracene.

\begin{tabular}{lccccccc}
\hline \hline Band type & $\begin{array}{c}1.0 \\
\text { beat }\end{array}$ & $\begin{array}{c}3.5 \\
\text { beat }\end{array}$ & $\begin{array}{c}4.5 \\
\text { beat }\end{array}$ & $\begin{array}{c}6.2 \\
\text { beat }\end{array}$ & $\begin{array}{c}9.7 \\
\text { beat }\end{array}$ & $\begin{array}{c}10.7 \mathrm{GHz} \\
\text { beat }\end{array}$ & $\begin{array}{c}\text { Sum of } \\
\text { modulations }\end{array}$ \\
\hline $\begin{array}{l}a \text { type: } \\
\text { calculated } \\
\text { observed }\end{array}$ & +0.69 & +0.004 & +0.006 & +0.004 & +0.42 & +0.70 & +1.82 \\
$\begin{array}{c}\text { b type: } \\
\text { calculated } \\
\text { observed }\end{array}$ & -0.55 & $\cdots$ & $\cdots$ & $\cdots$ & +0.36 & +0.52 & +1.43 \\
$\begin{array}{c}\text { chype: } \\
\text { calculated }\end{array}$ & -0.74 & -0.07 & +0.07 & +0.01 & -0.15 & +0.13 & -0.99 \\
$\begin{array}{c}d \text { type: } \\
\text { calculated }\end{array}$ & -0.31 & +0.03 & +0.03 & -0.06 & -0.62 & -0.68 & -0.99 \\
\hline \hline
\end{tabular}

$1.5^{\circ}$. In addition, the square root of the sum of the squares of the fourth row, which sum was not fixed by the calculation, is found to be very close to 1 (i.e., 1.003). For each of the four types of the fluorescence bands the absolute modulation depths predicted from the calculated $C$ are presented in Table II along with the available experimental values. Again the experimental values are consistently lower (by $20 \%-30 \%$ ) than the calculated ones, which deviation probably arises from the same causes as those which give rise to the less than ideal total modulation depth of the $1750 \mathrm{~cm}^{-1}$ band. One may note that the calculated values in Table II can serve to aid in the identification of the $d$-type bands. In particular, the calculated value of -0.73 for $M_{d}\left(\omega_{12}\right)$ suggests that perhaps the band at $1521 \mathrm{~cm}^{-1}$ is $d$ type (see the decay in Fig. 14).

Just as for $E_{\text {vib }}=1380 \mathrm{~cm}^{-1}, H_{0}$ for $E_{\text {vib }}=1420$ $\mathrm{cm}^{-1}$ may be calculated using Eq. (3.1) of paper I. This results in

$$
\begin{aligned}
H_{0}^{1420}= & E_{0} I \\
& \pm\left(\begin{array}{rrrr}
3.23 & -0.28 & -4.24 & -1.86 \\
-0.28 & 1.70 & 0.29 & 1.82 \\
-4.24 & 0.29 & 7.57 & 0.94 \\
-1.86 & 1.82 & 0.94 & 3.70
\end{array}\right) .
\end{aligned}
$$

Again it must be noted that the distribution of signs for the off-diagonal elements in $H_{0}$ is not a unique one ${ }^{7}$ and that other distributions are not precluded by the quantum beat results. In any case, the magnitudes of the coupling matrix elements are of the most interest, so this ambiguity in sign is not particularly limiting.

\section{C. $E_{\mathrm{vlb}}=1409,1501$, and $1514 \mathrm{~cm}^{-1}$}

The 1380 and $1420 \mathrm{~cm}^{-1}$ excitation bands occur in a region of the excitation spectrum which contains a number of other prominent bands ${ }^{14}$; the ones at 1409 , 1501 , and $1514 \mathrm{~cm}^{-1}$. Given that the former two excitations give rise to dynamical behavior that is characteristic of restricted IVR, one might expect the latter three excitations to do so, as well. Previously published spectral evidence $^{15}$ has indeed indicated that restricted IVR influences the molecular dynamics at these energies. However, time-domain evidence has been lacking. Figures 15-17 provide such evidence for the 1409,1501 , and $1514 \mathrm{~cm}^{-1}$ excitations, respectively. In each figure the uppermost decay corresponds to detection of the $390 \mathrm{~cm}^{-1}$ band, which from spectroscopic considerations ${ }^{14}$ and in analogy with the decay behavior of this band for $E_{\mathrm{vib}}=1380$ and $1420 \mathrm{~cm}^{-1}$, should be $a$ type. Consistent with this, Fourier analysis of the three $390 \mathrm{~cm}^{-1}$ decays reveals that all beat components enter into the decays with +1 phases. On the other hand, each of the spectra arising from the three excitations also contain bands with different decays than the corresponding $390 \mathrm{~cm}^{-1}$ decay, the most notable differences being that some of the beat components appear with -1 phases. Examples of such decays are shown in the lower portions of Figs. 15-17. It is pertinent to note that the order of magnitude of the beat frequencies appearing in the decays of these figures is the same as for

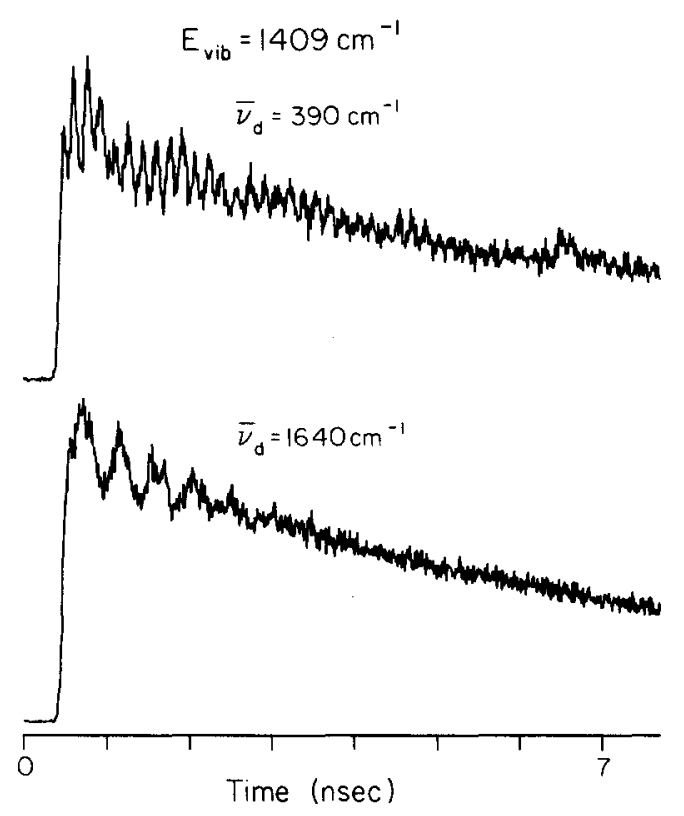

FIG. 15. Fluorescence decays of two bands in the $E_{\text {vib }}=1409 \mathrm{~cm}^{-1}$ spectrum of jet-cooled anthracene. The wave number shifts of the bands from the excitation energy are given in the figure. For the upper decay $R=16 \AA$, for the lower $R=1.6 \AA$. Note that the $390 \mathrm{~cm}^{-1}$ band is very isolated spectrally compared with the $1640 \mathrm{~cm}^{-1}$ band (see Ref. 15). Thus it was possible to use a relatively large value of $R$ in measuring the $390 \mathrm{~cm}^{-1}$ decay. The same holds true for the $390 \mathrm{~cm}^{-1}$ decays in Figs. 16 and 17. 


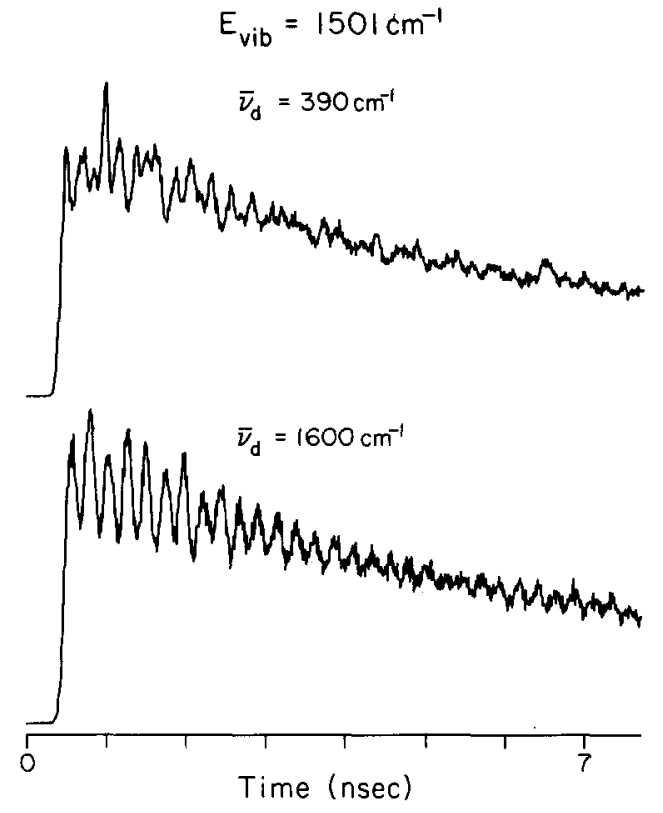

FIG. 16. Fluorescence decays of two bands in the $E_{\text {vib }}=1501 \mathrm{~cm}^{-1}$ spectrum of anthracene. The wave number shifts of the bands from the excitation energy are given in the figure. For the upper decay $R=24$ $\AA$, and for the lower one $R=1.6 \AA$.

the beat components associated with 1380 and $1420 \mathrm{~cm}^{-1}$ excitations.

It is clear from the experimental results that the decay behaviors of the 1409,1501 , and $1514 \mathrm{~cm}^{-1}$ excitations are qualitatively similar to the behaviors of the 1380 and $1420 \mathrm{~cm}^{-1}$ excitation bands, and, moreover, are consistent with the manifestations of restricted IVR. Being that the vibrational density of states (see Sec. VI A below) does not change much more than a factor of two in going from $E_{\mathrm{vib}}=1380$ to $E_{\mathrm{vib}}=1514 \mathrm{~cm}^{-1}$, this similarity is not particularly surprising. The significance

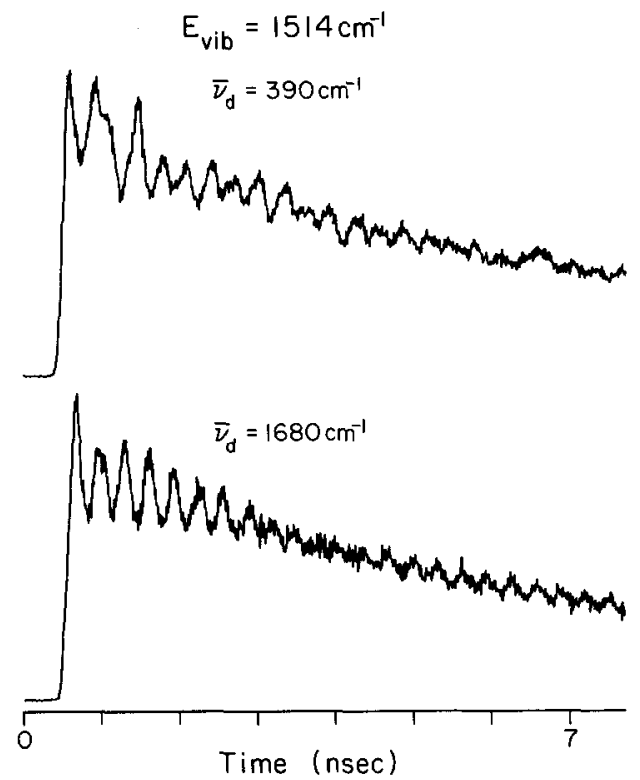

FIG. 17. Fluorescence decays of two bands in the $E_{\text {vib }}=1514 \mathrm{~cm}^{-1}$ spectrum of anthracene. The wave number shifts of the bands from the excitation energy are given in the figure. For the upper decay $R=32$ $\AA$, and for the lower one $R=2.4 \AA$. of the results is that they show the general occurrence of phase-shifted beats in the energy region corresponding to restricted IVR.

\section{HIGH ENERGY: $E_{\text {vib }}=1792 \mathrm{~cm}^{-1}$}

Above $E_{\mathrm{vib}}=1514 \mathrm{~cm}^{-1}$ in $S_{1}$ anthracene, there are only a small number of excitation energies which yield dispersed fluorescence spectra with resolvable (or partially resolvable) structure ${ }^{5,15}$ and which are thereby amenable to the direct study of IVR through the measurement of the temporal evolution of individual fluorescence band types. One such excitation corresponds to $E_{\text {vib }}=1792$ $\mathrm{cm}^{-1}$, which is most likely a combination band of the $385 \mathrm{~cm}^{-1}\left(S_{1} 12\right)$ mode $^{14}$ with the $1409 \mathrm{~cm}^{-1}$ band. As may be seen from Fig. 18, the dispersed fluorescence spectrum which arises from this excitation is quite congested compared with lower energy spectra, but nevertheless has at least two discrete bands of weak intensity in the blue portion of the spectrum (the bands at 390 and $780 \mathrm{~cm}^{-1}$ ) and also some incompletely resolved structure with overall strong intensity in the region near the $S_{1}$ origin. The 390 and $780 \mathrm{~cm}^{-1}$ bands (vibrationally unrelaxed or a-type bands), being assignable in terms of optically active modes, correspond to transitions from the optically prepared zero-order state. The bands in the

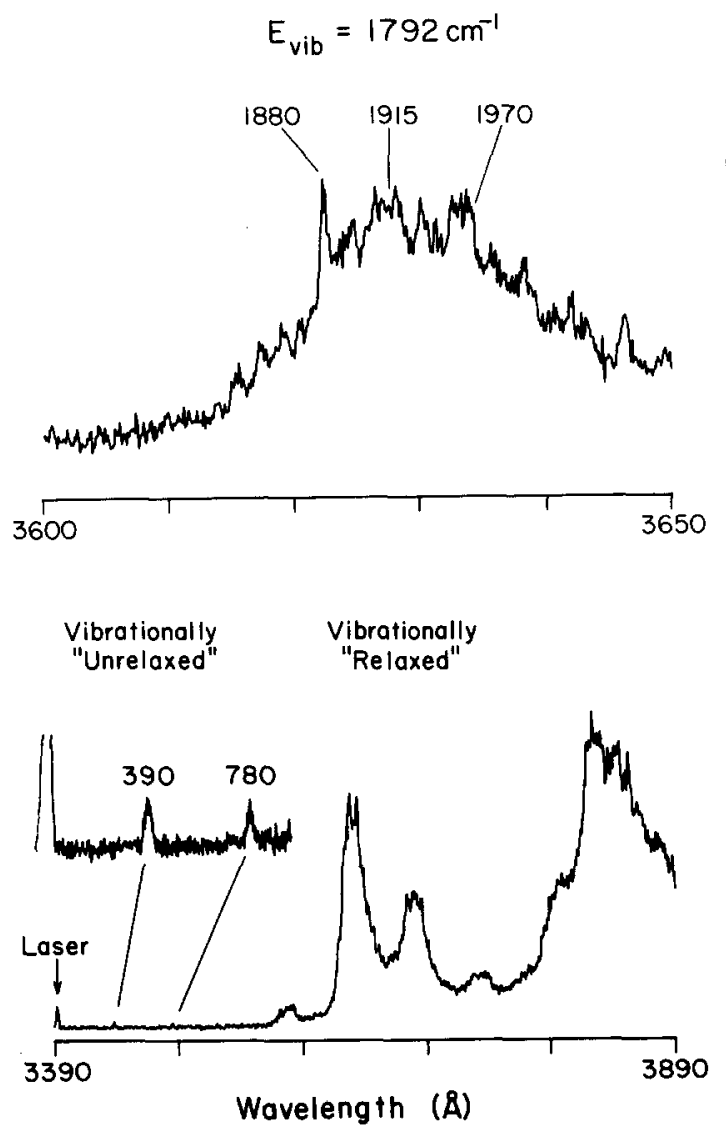

FIG. 18. Dispersed fluorescence resulting from excitation of jet-cooled anthracene to $S_{1}+1792 \mathrm{~cm}^{-1}$. The upper portion was taken with $R$ $=0.5 \AA$. For the lower portion $R=1.6 \AA$ for the main spectrum and $R=3.2 \AA$ for the inset, which corresponds to the vibrationally unrelaxed region. Various bands in the spectra are labeled with their wave number shifts from the excitation energy. 
congested region near the $S_{1}$ origin arise predominantly from zero-order states populated by the IVR process (vibrationally relaxed bands). Given that the two types of bands arise from different zero-order states, one expects differences in their decays to provide a picture of vibrational energy flow at this energy (see Sec. V B of I).

Figure 19 presents the decays of the 390 and 780 $\mathrm{cm}^{-1}$ bands. It is evident that they are very similar. Fitting each according to the procedure of Sec. II C gives a short component lifetime of $22 \mathrm{ps}$ and a long component lifetime of $6.4 \mathrm{~ns}$. Figure 20 shows a typical fit to the data. We would make two points about the lifetimes obtained from the fits. Firstly, the short lifetime differs significantly from the value of $\sim 75$ ps that we have quoted in a preliminary work. ${ }^{5}$ This discrepancy arises from the fact that in the previous work we fit only the initial portion of a given decay to a single exponential. Evidently, the contribution of the long time component at early time is substantial enough to render such a procedure inaccurate. One expects the double exponential fitting procedure to yield a much more accurate fast time constant. Secondly, the long time component has a lifetime that matches the lifetime ${ }^{15}$ of the total fluorescence decay at this energy, as one would expect from Eq. (5.7) of paper I. Finally, it is worth noting that Fourier analysis (see Fig. 21) reveals that all of the large number of beat components which contribute to the decays of Fig. 19 have +1 phases as expected for an $a$-type band. (For

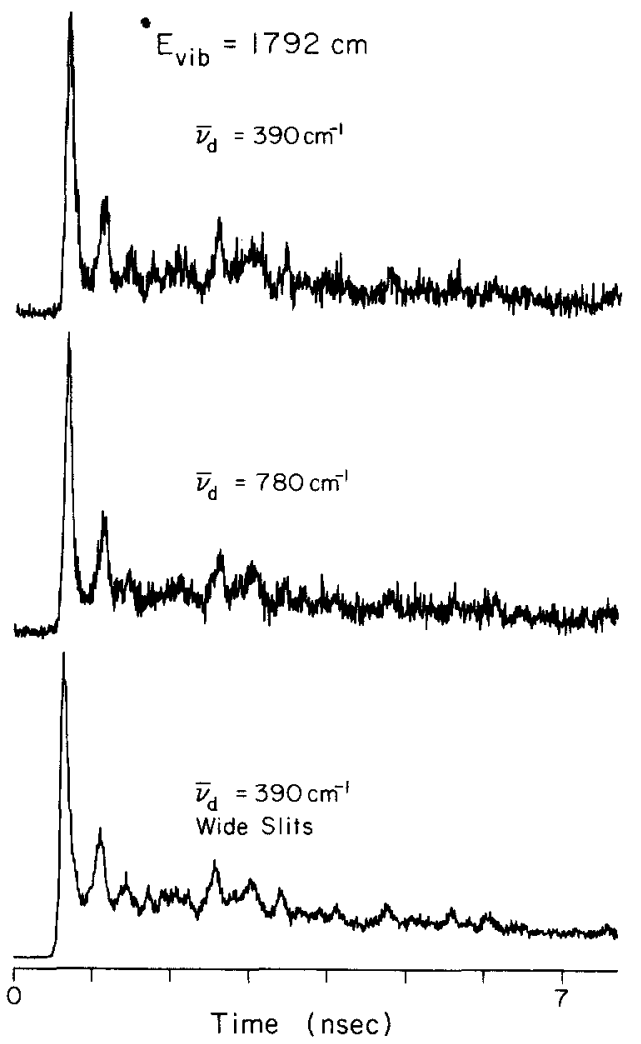

FIG. 19. Fluorescence decays for the 390 and $780 \mathrm{~cm}^{-1}$ bands in the spectrum of anthracene excited to $S_{1}+1792 \mathrm{~cm}^{-1}$. The top two decays were taken with $R=3.2 \AA$. For the bottom decay $R=32 \AA$. A slight increase in the relative intensity of the long time component is apparent in the lower decay compared with the upper two.

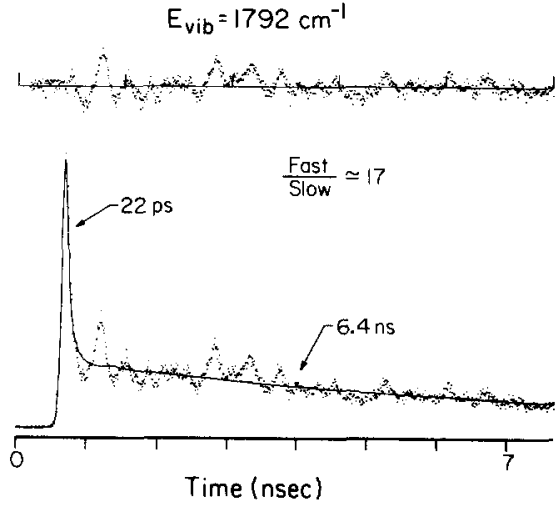

FIG. 20. Double exponential fit (line) to the measured decay of Fig. 19 (bottom). The weighted difference (residual) between the observed decay and the fitted curve appears at the top of the figure. Best fit values of the two lifetimes and the ratio of fast to slow fluorescence are given in the figure. One notes that the best fit fast to slow ratio is greater than what one would judge by eye from the decay because the finite temporal response of detection tends to reduce the apparent magnitude of the fast component.

comparison we include in Fig. 21 the uncluttered Fourier spectrum of the $a$-type $390 \mathrm{~cm}^{-1}$ decay corresponding to $S_{1}+1380 \mathrm{~cm}^{-1}$ excitation.)

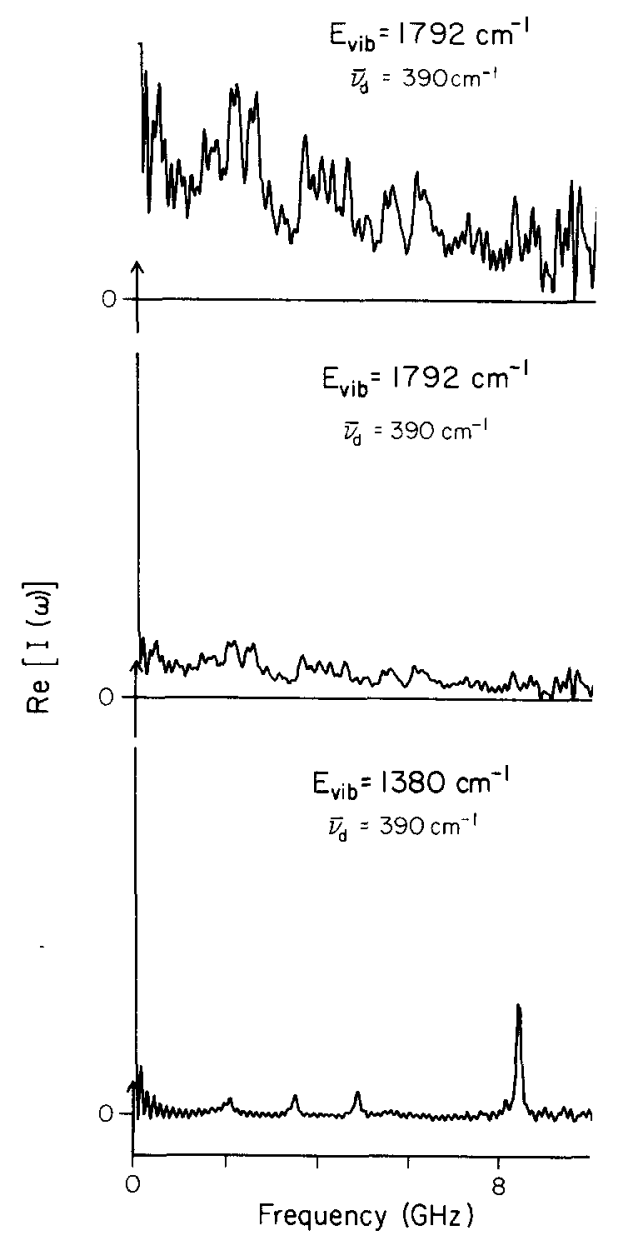

FIG. 21. Top and middle: Fourier spectra $[I(\omega)-$ Sec. II B] of the decay of Fig. 19 (bottom). The top spectrum is the same as the middle one but is expanded along the ordinate. Bottom: The Fourier spectrum of the decay of the $390 \mathrm{~cm}^{-1}$ band in the $E_{\mathrm{vib}}=1380 \mathrm{~cm}^{-1}$ spectrum [see Fig. 4 (bottom)], presented to provide an idea of the kind of Fourier "structure" (note the oscillations) to be expected as a result of the truncation of measured decays. 
Since the 390 and $780 \mathrm{~cm}^{-1}$ bands are weak in intensity and occur in a region close to the excitation wavelength, several checks were made to ensure that the measured decays of these bands represent molecular decays and not scattered laser light. These checks included measuring decays at a detection wavelength of $3410 \AA$ [Fig. 22(a)], a wavelength which is spectrally closer to the exciting light but at which there is no fluorescence, and measuring decays without any anthracene in the free-jet expansion [Fig. 22(b)]. These checks yielded no evidence for any significant scatter contribution. Some leakage light was found to contribute to the decays. However, this spurious component was determined to be due to molecular fluorescence and to contribute to the long time intensity of the decays. This is evident from Fig. 19 (bottom), which shows an increased intensity of the long time component of the $390 \mathrm{~cm}^{-1}$ decay for decreased spectral resolution of detection relative to that used to detect the decay of Fig. 19 (top). Laser-to-nozzle distance and carrier gas dependences were also checked in order to assess the influence of intermolecular interactions on

a)
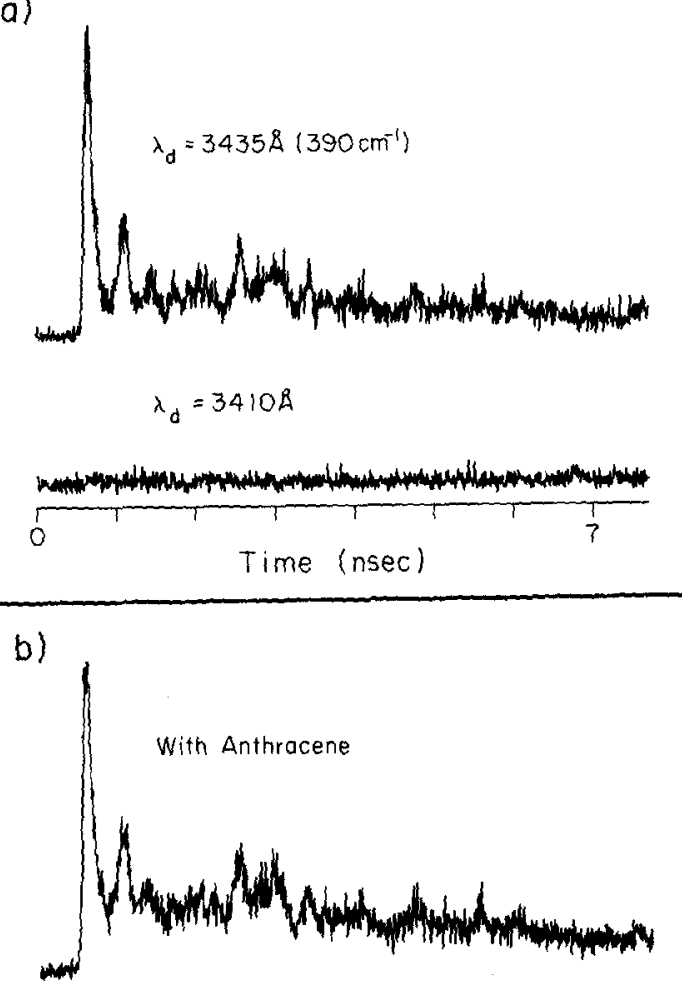

Without Anthracene

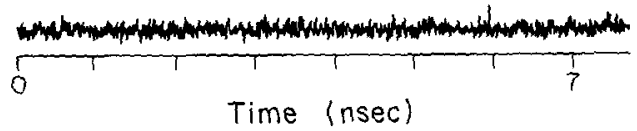

FIG. 22. (a) Decays measured for similar collection times for onresonance (top) and off-resonance (bottom) detection, and for excitation of anthracene to $E_{\mathrm{v} i b}=1792 \mathrm{~cm}^{-1}$. Conditions for both decays $(R=3.2$ $\AA$ ) were identical except for detection wavelength. (b) Decays measured for similar collection times and under the same experimental conditions (excitation to $E_{v i b}=1792 \mathrm{~cm}^{-1}$, detection of the $390 \mathrm{~cm}^{-1}$ band with $R$ $=3.2 \AA$ ) except that the bottom decay corresponds to a situation wherein the free-jet expansion was devoid of anthracene.

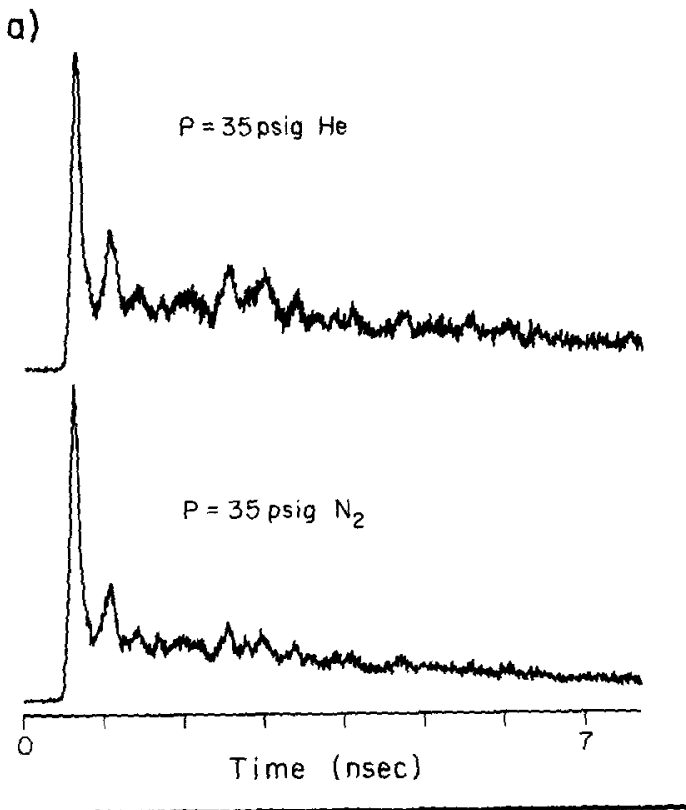

b)

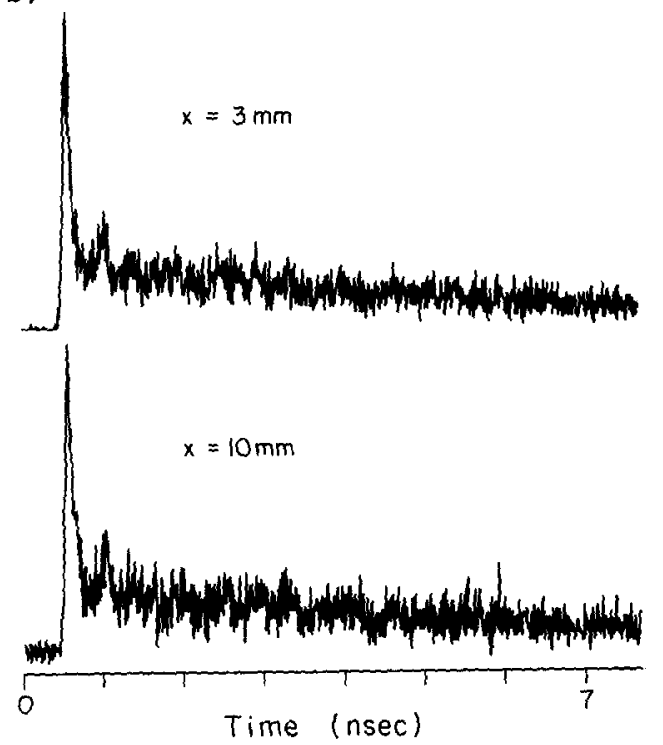

FIG. 23. Fluorescence decays of the $390 \mathrm{~cm}^{-1}$ band in the $E_{\text {vib }}=1792$ $\mathrm{cm}^{-1}$ spectrum of anthracene for (a) different carrier gas conditions and (b) different laser to nozzle distances. For the decays in (a), $R=32 \AA$. In (b), $R=16 \AA$.

the observations. No significant effects of this kind were observed (see Fig. 23).

Figure 24 presents decays corresponding to three fluorescence detection wavelengths in the region of the $S_{1}$ origin. There are several notable features of these decays. Firstly, each has a time constant of $\sim 6 \mathrm{ns.}$ Secondly, all of the decays are modulated, but exhibit different beat patterns. Finally, the decays all have finite rise times. As may be seen from Fig. 24 (bottom), which is a decay measured at the same detection wavelength as used for the decay above but at lower spectral resolution, there is a pronounced spectral resolution effect on the decays measured in this region. Given a number of different types of fluorescence bands in the same spectral region, this is not surprising. ${ }^{7}$ The apparent rise times of 


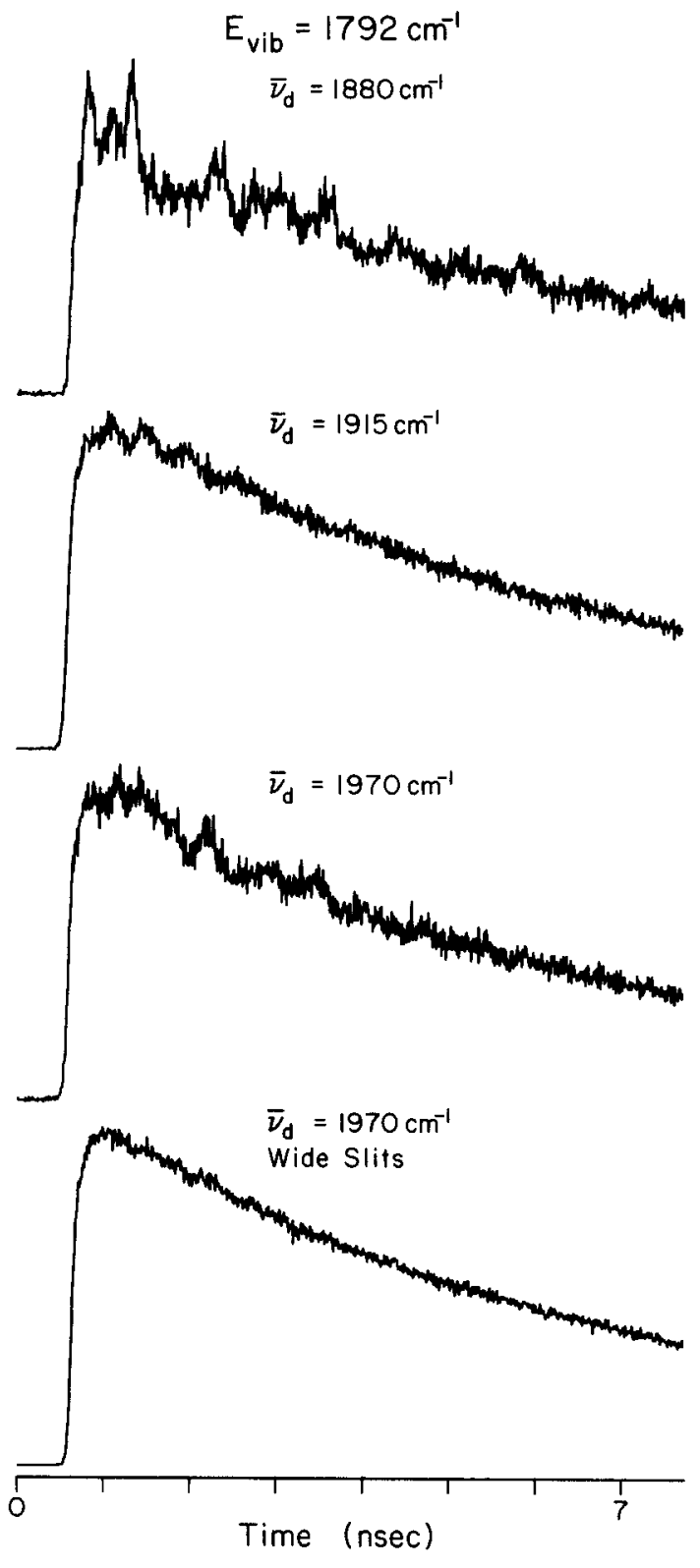

FIG. 24. Fluorescence decays of bands in the vibrationally relaxed region of the $E_{\mathrm{vib}}=1792 \mathrm{~cm}^{-1}$ spectrum of anthracene [see Fig. 18 (top)]. Wave number shifts of the detected bands from the excitation energy are given in the figure. From top to bottom, $R=1.6,3.2,1.6$, and $16 \AA$.

the decays were examined in several ways to determine whether they were real rise times. For example, all of the decays were compared with decays of the $390 \mathrm{~cm}^{-1}$ band measured immediately before or immediately after. In all cases the decays of Fig. 24 peaked after the $390 \mathrm{~cm}^{-1}$ decay did and appeared to rise at the same rate at which the short component of the $390 \mathrm{~cm}^{-1}$ transient decayed. Also, the smooth decay of Fig. 24 (bottom), and others like it, were compared with calculated decays of $6 \mathrm{~ns}$ exponentials convoluted with the measured system response function. In all cases a significant difference between the calculated and measured decays was found, with the measured decays rising more slowly than the calculated ones. (Notably, this difference was not found for fluorescence at the same detection wavelength when the excitation was to $E_{\mathrm{vib}}=2750 \mathrm{~cm}^{-1}$.) Finally, Fourier analysis of the decays reveals Fourier components with negative phases. Such components must be present for a decay with a rise time. Notably, no components with negative phases modulate the 390 or $780 \mathrm{~cm}^{-1}$ decays.

We interpret the results for $E_{\mathrm{vib}}=1792 \mathrm{~cm}^{-1}$ in terms of dissipative vibrational energy flow. The 390 and $780 \mathrm{~cm}^{-1} a$-type decays, being as they are direct representations of the $|a\rangle$ content of the excited state (see Sec. V of I), show that vibrational energy redistributes dissipatively from $|a\rangle$ to the states coupled to $|a\rangle$ on a time scale of 22 ps. This initial redistribution of energy is also reflected in the risetimes of the decays of the bands near the $S_{1}$ origin, decays which are direct representations of the contributions of the states other than $|a\rangle$ to the excited state. ${ }^{7}$ The presence of beats in the fluorescence decays is a manifestation of the fact that although the initial energy flow is substantially irreversible, it does not reach a steady state on the $22 \mathrm{ps}$ time scale. Rather, the vibrational content of the excited state continues to evolve to some extent throughout the time scale of the fluorescence lifetime.

Regarding the number $N$ of coupled vibrational levels being manifested in the fluorescence behavior of the $S_{1}+1792 \mathrm{~cm}^{-1}$ excitation, it is more difficult to determine an accurate value than it is in the lower energy regime. This is a result of the large number of beat components in and complicated Fourier spectra of the $1792 \mathrm{~cm}^{-1}$ decays. Nevertheless, it is reasonably certain from the Fourier transform of the $390 \mathrm{~cm}^{-1}$ decay (Fig. 21) that greater than 10 coupled levels give rise to the observed results. This is supported by the parameters derived from the fits to the decays of Fig. 19, since from these it is found that the ratio of fast to slow fluorescence in the decays is $\sim 17$. This ratio represents a lower limit to the number of coupled states in the dissipative regime (see Sec. V B of paper I). It is notable that if one assumes that the number of coupled levels at a particular $E_{\mathrm{vib}}$ scales with the vibrational density of states (see Sec. VI A below), then the roughly fivefold increase in this density in going from 1400 to $1800 \mathrm{~cm}^{-1}$, along with the values of $N$ at 1380 and $1420 \mathrm{~cm}^{-1}$, would suggest that there are 15-20 states coupled at $E_{\text {vib }}=1792 \mathrm{~cm}^{-1}$.

The results for $E_{\mathrm{vib}}=1792 \mathrm{~cm}^{-1}$ are also interpretable in terms of the dense intermediate case of radiationless transitions. ${ }^{18}$ The vibrational coupling at this energy is such that the number of effectively coupled zero-order states $N$ is larger than in the lower energy regime of restricted IVR (sparse intermediate case), but smaller than would be the case if the intrinsic linewidths of the states exceeded their energy spacings (statistical limit). This conclusion is reached by consideration of two facets of the fluorescence decays. Firstly, the short-time component of the 390 and $780 \mathrm{~cm}^{-1}$ decays and the rise times of the other decays are indicative of an intramolecular dephasing between the eigenstates composing the optically prepared state. Secondly, the presence in all of the decays of a long-time component with beats indicates that the dephasing does not preclude partial recurrences on the time scale of the fluorescence lifetime. The short- 
time behavior distinguishes the dense from the sparse intermediate case, while the presence of long-lived intramolecular coherence distinguishes the intermediate case from the statistical limit. ${ }^{18}$

\section{DISCUSSION AND CONCLUSIONS}

\section{A. IVR and coherence vs vibrational energy}

The experimental results presented above allow one to make a number of different conclusions concerning IVR in anthracene. The first pertain to the nature of the process as a function of vibrational energy in the molecule. It is clear in light of the discussion in Sec. V B of $I$, that the low, intermediate, and high energy results for anthracene represent manifestations of absent, restricted, and dissipative IVR, respectively. In the low energy regime ( $\leq 1200 \mathrm{~cm}^{-1}$ ), vibrational mixing is not extensive, if present at all. The excitation process prepares just one vibrational eigenstate which undergoes no further vibrational evolution. For somewhat higher energies (i.e., 1380$1520 \mathrm{~cm}^{-1}$ ) the vibrational coupling becomes appreciable; systems of coupled states involving two to approximately 10 levels characterize the regime. Upon preparation of an optically active zero-order state, IVR occurs but is restricted in the sense that essentially full recurrences in the vibrational energy distribution occur on the timescale of the fluorescence lifetime. The timescale of IVR in the restricted regime is given by the order of magnitude of the inverses of the beat frequencies which modulate dispersed fluorescence decays. For anthracene the beat frequencies are on the order of $5 \mathrm{GHz}$, leading to a time scale for restricted IVR of several hundreds of picoseconds. Finally, at high energies (e.g., $E_{\text {vib }}=1792 \mathrm{~cm}^{-1}$ ) the extent of vibrational coupling is great enough (systems of $\geq 10$ levels) that dissipative IVR occurs. In these cases, IVR is such that nothing close to a full recurrence occurs on the fluorescence time scale. The time scale of IVR in the dissipative regime is given by that of the initial, approximately exponential dephasing of the optically prepared state. For the $E_{\mathrm{vib}}=1792 \mathrm{~cm}^{-1}$ band, $\tau_{\mathrm{IVR}}$ $\simeq 22$ ps.

It is of interest to correlate changes in the nature of IVR in anthracene with the vibrational density of states $\left(\rho_{\text {vib }}\right)$ in the $S_{1}$ manifold. We have calculated $\rho_{\text {vib }}$ at various energies using a direct count procedure and the calculated frequencies of Refs. 19. The results are shown in Fig. 25. In the region of no IVR, $\rho_{\text {vib }}$ is less than $\simeq 10$ per wave number. For $E_{\text {vib }}=1380-1520 \mathrm{~cm}^{-1}, \rho_{\text {vib }}$ goes from $\sim 25-40$ per $\mathrm{cm}^{-1}$. And for $E_{\text {vib }}=1800 \mathrm{~cm}^{-1}, \rho_{\text {vib }}$ $\simeq 120$ per $\mathrm{cm}^{-1}$. These values of $\rho_{\text {vib }}$ for the different regimes of IVR in anthracene are useful for two reasons. Firstly, they suggest that vibrational coupling in the molecule is selective. For instance, at $E_{\mathrm{vib}}=1380 \mathrm{~cm}^{-1}$ there are three strongly coupled vibrational states within an $8.4 \mathrm{GHz}$ energy interval. But, if there were mixing between all the states in this interval, then since $\rho_{\text {vib }}$ $=25$ per $\mathrm{cm}^{-1}$ one would expect at least twice as many coupled levels. A similar point can be made for the $E_{\text {vib }}$ $=1420$ and $1792 \mathrm{~cm}^{-1}$ excitations; the number of coupled levels is less than would be expected from consideration

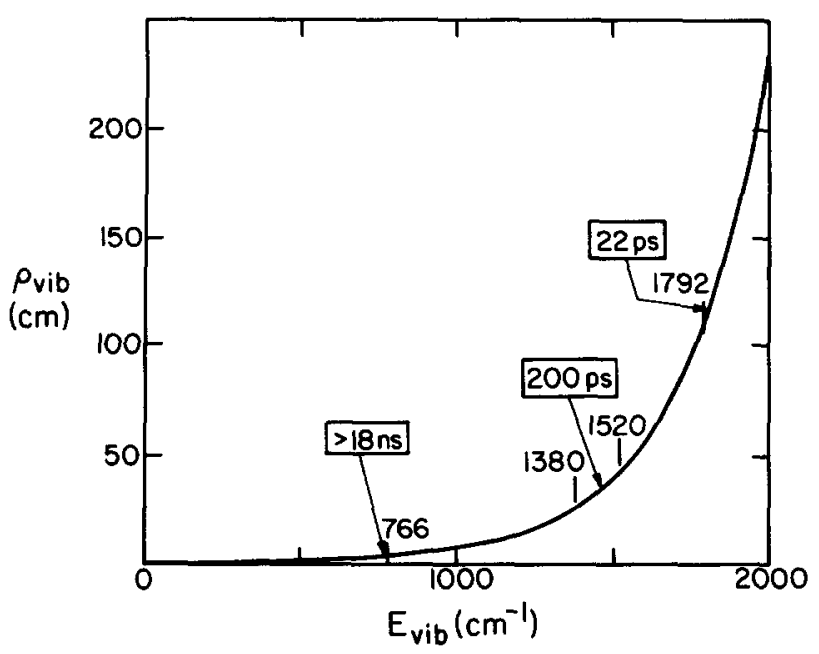

FIG. 25. Vibrational density of states $\left(\rho_{\text {vib }}\right)$ vs vibrational energy $\left(E_{\text {vib }}\right)$ in anthracene calculated using a direct count method and the frequencies of Refs. 19. Given in the figure are characteristic times for IVR in the absent, restricted, and dissipative regimes.

of the total density of vibrational states. This selectivity in coupling is not unreasonable. For vibrational states to be coupled by anharmonic interactions, they must have the same vibrational symmetry. In $D_{2 h}$ anthracene this means that a given vibrational state can couple anharmonically with about one-eighth of the total number of other states in the molecule. Thus, selectivity in coupling is expected on the basis of symmetry considerations. Secondly, the specific values for $\rho_{\text {vib }}$ are useful for their predictive value. Based on these values, symmetry considerations, and knowledge of $\rho_{\text {vib }}$ vs energy, one can make predictions of the nature of IVR as a function of energy for other molecules.

A last point of interest concerning IVR as a function of excess energy in anthracene derives from the connection between IVR behavior and quasiperiodic or chaotic vibrational dynamics in molecules. ${ }^{20,21}$ In a classical system, the loss of coherence reflects the degree of chaos in that system. For the dynamics in real molecules, however, one must consider quantum chaos, the criteria for the existence of which and the relationship to spectroscopic observables of which are not fully understood. These points have been discussed in a number of papers. The review articles by Rice $^{20}$ and by Noid $e t$ al. ${ }^{21}$ are recommended as being fuller treatments of the subject. Here, we have no intention of suggesting that our experimental results prove the presence or absence of quantum chaos in anthracene, but instead that they may be interpreted in terms of one particular indicator of quantum chaotic behavior. ${ }^{22}$

As discussed by Rice, ${ }^{20}$ some of the dynamical properties discovered in simple model systems can be important in understanding the nature of intramolecular vibrational "relaxation." Recently, for a model Hamiltonian (Henon-Heiles), the transition with increasing energy from classical mechanical quasiperiodic to chaotic vibrational dynamics has been found ${ }^{22}$ to be correlated with quantum mechanical quantities derived from the same Hamiltonian. Regular, periodic behavior of the quantity 


$$
\rho_{\gamma}(t) \equiv|\langle\Psi(t) \mid \gamma\rangle|^{2},
$$

which is the contribution of the harmonic state $|\gamma\rangle$ to the time dependent vibrational wavefunction $|\Psi(t)\rangle$, correlates with classical quasiperiodic behavior. On the other hand, irregular behavior of $\rho_{\gamma}(t)$ correlates with classical chaotic behavior. Since

$$
\rho_{\gamma}(t) \sim I_{\gamma}(t)
$$

where $I_{\gamma}(t)$ is the intensity vs time of a $\gamma$-type fluorescence band (see Sec. V A, paper I), then in the sense of Refs. 22 the decays of fluorescence are indicators of quasiperiodic or chaotic vibrational dynamics. The regular, periodic decays characterizing the restricted IVR regime in anthracene suggest quasiperiodic quantum dynamics. The less regular, high energy dissipative decays suggest quantum chaotic behavior. Regarding this last point, we would note that it must be made with some caution, since the fluorescence lifetime of the molecule restricts the time scale over which recurrences may be observed. Thus, as pointed out by the referee of this paper, if the coupling giving rise to dissipative IVR at say $E_{\mathrm{vib}}=1792 \mathrm{~cm}^{-1}$ in $S_{1}$ anthracene were to occur instead in the $S_{0}$ state, then the longer lifetime associated with infrared emission might make possible the observation of essentially full recurrences-indicating quasiperiodic dynamics-in the "dissipative" decay. From this it is clear that (1) we must qualify our use of the term dissipative to mean "dissipative on the time scale of the fluorescence lifetime of the molecule" and (2) we must recognize that fluorescence decay behavior as an indicator of chaotic dynamics is ultimately limited by the time scale of the experiment. As for the case of restricted IVR, where full recurrences are observed experimentally, the important point is that one is observing the coherent evolution of the vibrational wave packet in the molecule.

\section{B. Coupling matrix elements}

Besides providing information concerning the nature and timescale of IVR in anthracene, the experimental results also provide information pertaining to the details of the couplings which give rise to IVR. Thus, for the restricted IVR that occurs for $E_{\text {vib }}=1380$ and 1420 $\mathrm{cm}^{-1}$, one can see from Eqs. (4.3) and (4.6) that the magnitudes of the coupling matrix elements between zero-order states range from 0.3 to $4.2 \mathrm{GHz}$. For the dissipative $1792 \mathrm{~cm}^{-1}$ case, the variance in coupling matrix elements, $\bar{V}$, can be calculated ${ }^{23}$ by using the golden rule-type expression $1 / \tau_{\mathrm{IVR}} \simeq 2 \pi \bar{V}^{2} \rho$ and by assuming that because of symmetry selectivity in coupling, $\rho=\rho_{\text {vib }} / 8=15$ per $\mathrm{cm}^{-1}(0.5$ per $\mathrm{GHz})$. This gives $|\bar{V}|$ $=3.8 \mathrm{GHz}$. It is clear therefore that coupling matrix elements on the order of several $\mathrm{GHz}$ heavily influence both restricted and dissipative IVR in the molecule.

Another point concerning the vibrational coupling elements in anthracene pertains to the distribution of the coupling between zero-order states. One can see from Eqs. (4.3) and (4.6) that significant coupling occurs between all the coupled zero-order states. Thus, for instance, one would be mistaken in viewing the IVR process as arising from the coupling of an optically active state with a manifold of uncoupled bath states. There is coupling within the bath manifold, and this coupling affects the IVR dynamics.

\section{The purity of optically prepared states}

As we have discussed in Sec. IV of I, various experimental limitations can prevent the complete characterization of IVR processes by time-resolved spectroscopy. One limitation of a fundamental nature involves the inability to optically prepare all the eigenstates that arise from the coupling of a given set of zero-order states. In cases where this limitation applies, the optically prepared state is not a pure zero-order harmonic vibrational state. At the very outset the vibrational energy is distributed amongst a number of vibrational motions.

Under the conditions of our experiments, we are able to coherently prepare eigenstates within a range of approximately $2 \mathrm{~cm}^{-1}$. Therefore, any coupling interactions on this order will prevent the preparation of a pure zero-order state. There is experimental evidence that vibrational couplings of this magnitude are present in anthracene. Firstly, the sums of the absolute quantum beat modulation depths of the non-a-type fluorescence bands for $E_{\text {vib }}=1380$ and $1420 \mathrm{~cm}^{-1}$ are not -1 . This kind of behavior can be the result of the inability to prepare a pure zero-order state (see Sec. IV A of I). A second point related to the first is that the absolute modulation depths calculated from the eigenvector matrices (4.2) and (4.5) are consistently greater in magnitude than observed modulation depths. This too, can be due to an impure initial state. Finally, evidence from excitation spectra ${ }^{16}$ indicates that Fermi resonances that give rise to splittings on the order of several $\mathrm{cm}^{-1}$ are prevalent in anthracene.

All of this evidence points to the fact that one must be' somewhat cautious in determining the purity of the $|a\rangle$ state. For anthracene, it probably is the case in the restricted regime that impure initial states are prepared by the laser, although the sums of absolute modulation depths for non- $a$-type bands are close enough to -1 to suggest that the degree of impurity is not particularly great.

\section{Spectral vs temporal measurements}

In light of the previous section, it is pertinent to discuss the merits of time-resolved vs time-integrated approaches to the experimental study of IVR and vibrational coupling. On the one hand, we have seen that the limitations of finite excitation bandwidth and finite temporal resolution can restrict the utility of time-resolved techniques to the regime of relatively small magnitude matrix elements. In the cases of large magnitude matrix elements, time-integrated fluorescence excitation spectra can provide valuable information about long range coupling that is unavailable from fluorescence decays. On the other hand, time-resolved measurements have many advantages over fluorescence excitation and dispersed fluorescence studies. Medium resolution $\left(\sim 0.05 \mathrm{~cm}^{-1}\right)$ 
excitation spectra are only useful for the study of couplings with matrix elements on the order of, or greater than, rotational contour widths (one to several wave numbers for jet-cooled large molecules). For smaller couplings, rotational congestion necessitates taking Doppler-free excitation spectra to obtain the number and distribution of the eigenstates resulting from the coupled system of zeroorder levels. Whatever the magnitudes of the matrix elements, it is never certain from excitation spectra alone, that the structure in them arises from a coupled system of levels. This is in contrast to time-resolved measurements which, by virtue of quantum beat phase behavior, can differentiate between the manifestations of coupled vs uncoupled states.

Time-integrated dispersed fluorescence measurements also have limited utility in the study of IVR. Such measurements cannot provide detailed information pertaining to the evolution of vibrational energy in a molecule. For instance, the dispersed fluorescence spectra arising from excitations into the restricted IVR regime look very much the same as the spectra resulting from excitation into the dissipative IVR regime. It is only with knowledge from time-resolved studies that one can confidently make conclusions about the nature of IVR behavior. Also, in determining time scales for IVR, dispersed fluorescence measurements are limited. There is no way by such measurements to determine the recurrence times characterizing vibrational energy flow in the restricted regime. In the dissipative regime, one can in principle determine the time scale for IVR by measurements of the relative intensities of vibrationally relaxed and unrelaxed bands in a given spectrum. The problem with such measurements is that both týpes of fluorescence occur together in the congested region at and to the red of the $0_{0}^{0}$ energy. There is no accurate way to separate out the two types of contributions in this region unless one has precise knowledge of the emission Frank-Condon factors for the optically prepared state.

\section{E. Future directions}

The results and conclusions of this paper point to several areas wherein further work could add significantly to the understanding of IVR in anthracene in particular, and large molecules in general. One such area is the role of rotations. In the following paper we show how the rotational level structure can affect anharmonic interactions.

A second area involves the question of the significance of large magnitude matrix elements in the vibrational coupling of large molecules. As we have mentioned earlier, work directed toward this problem is in progress. ${ }^{16}$
A final area involves the determination of the $S_{1}$ vibrational normal modes of anthracene, with the aim of ultimately trying to make the connection between observed coupling matrix elements and the natures of the vibrational states that are coupled. One wonders if all zero-order states of a given symmetry within a given energy interval are significantly coupled, or if, on the other hand, there are propensity rules governing the magnitudes of the couplings between various types of vibrations. Such knowledge is of obvious importance to the understanding of both IVR and vibrational level-specific dynamics.

\section{ACKNOWLEDGMENTS}

This work was supported by the National Science Foundation through Grant No. DMR-8105034.

' W. R. Lambert, P. M. Felker, and A. H. Zewail, J. Chem. Phys. 75, 5958 (1981).

${ }^{2}$ W. R. Lambert, P. M. Felker, and A. H. Zewail, J. Chem. Phys. 81, 2217 (1984).

${ }^{3}$ P. M. Felker and A. H. Zewail, Chem. Phys. Lett. 102, 113 (1983).

${ }^{4}$ P. M. Felker and A. H. Zewail, Phys. Rev. Lett. 53, 501 (1984).

${ }^{5}$ P. M. Felker and A. H. Zewail, Chem. Phys. Lett. 108, 303 (1984).

${ }^{6}$ See, for example: C. Lewis, W. R. Ware, L. J. Doemeny, and T. L. Nemzek, Rev. Sci. Instrum. 44, 107 (1973).

${ }^{7}$ P. M. Felker and A. H. Zewail, J. Chem. Phys. 82, 2961 (1985).

${ }^{8}$ W. Kaplan, Advanced Calculus (Addison-Wesley, Reading, Mass., 1973), pp. 512-14.

${ }^{9}$ (a) J. W. Cooley and J. W. Tukey, Math. Comput. 19, 297 (1965); (b) E. O. Brigham, The Fast Fourier Transform (Prentice-Hall, Englewood Cliffs, 1974).

${ }^{10}$ E. Bartholdi and R. R. Ernst, J. Magn. Reson. 2, 9 (1973).

${ }^{1}$ P. M. Felker and A. H. Zewail, J. Chem. Phys. 82, 2994 (1985).

${ }^{12}$ P. R. Bevington, Data Reduction and Error Analysis for the Physical Sciences (McGraw-Hill, New York, 1969).

${ }^{13}$ J. Chaiken, M. Gurnick, and J. D. McDonald, J. Chem. Phys. 74, 106 (1981).

${ }^{14}$ W. R. Lambert, P. M. Felker, J. A. Syage, and A. H. Zewail, J. Chem. Phys. 81, 2195 (1984).

${ }^{15}$ W. R. Lambert, P. M. Felker, and A. H. Zewail, J. Chem. Phys. 81, 2209 (1984).

${ }^{16}$ B. W. Keelan and A. H. Zewail J. Chem. Phys. 82, 3011 (1985).

${ }^{17}$ This interpretation is further supported by results reported in Ref. 16, which show that there are indeed two overlapping rotational band contours separated by about one wave number in the $1380 \mathrm{~cm}^{-1}$ region of the anthracene excitation spectrum.

${ }^{18}$ K. F. Freed and A. Nitzan, J. Chem. Phys. 73, 4765 (1980).

${ }^{19}$ (a) D. J. Evans and D. B. Scully, Spectrochim. Acta 20, 891 (1964); (b) B. N. Cyvin and S. J. Cyvin, J. Phys. Chem. 73, 1430 (1969). Note that the values reported in these papers represent those calculated for $S_{0}$ modes. One expects that the $S_{1}$ values are lower on the average than these. Therefore, in reality the $\rho_{\text {vib }}$ vs $E_{\text {vib }}$ curve of Fig. 25 probably is shifted somewhat toward lower energies.

${ }^{20}$ For an overview see: S. A. Rice, Advances in Laser Chemistry, edited by A. H. Zewail (Springer, New York, 1978), p. 2.

${ }^{21}$ For a review see: D. W. Noid, M. L. Koszykowski, and R. A. Marcus, Annu. Rev. Phys. Chem. 32, 267 (1981).

${ }^{22}$ For example: (a) G. Hose and H. S. Taylor, Chem. Phys. 84, 375 (1984); (b) M. D. Feit and J. A. Fleck, Jr., J. Chem. Phys. 80, 2578 (1984).

${ }^{23}$ C. Tric, Chem. Phys. 14, 189 (1976). 